\title{
Chronic Intermittent Ethanol Exposure Enhances the Excitability and Synaptic Plasticity of Lateral Orbitofrontal Cortex Neurons and Induces a Tolerance to the Acute Inhibitory Actions of Ethanol
}

\author{
Sudarat Nimitvilai', Marcelo F Lopez ${ }^{2}$, Patrick J Mulholland ${ }^{1,2}$ and John J Woodward ${ }^{*, 1,2}$ \\ 'Department of Neuroscience, Medical University of South Carolina, Charleston, SC, USA; ${ }^{2}$ Addiction Sciences Division, Department of Psychiatry \\ and Behavioral Sciences, Medical University of South Carolina, Charleston, SC, USA
}

\begin{abstract}
Alcoholism is associated with changes in brain reward and control systems, including the prefrontal cortex. In prefrontal areas, the orbitofrontal cortex (OFC) has been suggested to have an important role in the development of alcohol-abuse disorders and studies from this laboratory demonstrate that OFC-mediated behaviors are impaired in alcohol-dependent animals. However, it is not known whether chronic alcohol (ethanol) exposure alters the fundamental properties of OFC neurons. In this study, mice were exposed to repeated cycles of chronic intermittent ethanol (CIE) exposure to induce dependence and whole-cell patch-clamp electrophysiology was used to examine the effects of CIE treatment on lateral OFC (IOFC) neuron excitability, synaptic transmission, and plasticity. Repeated cycles of CIE exposure and withdrawal enhanced current-evoked action potential (AP) spiking and this was accompanied by a reduction in the afterhyperpolarization and a decrease in the functional activity of SK channels. CIE mice also showed an increase in the AMPA/NMDA ratio, and this was associated with an increase in GluAI/GluA2 AMPA receptor expression and a decrease in GluN2B NMDA receptor subunits. Following CIE treatment, IOFC neurons displayed a persistent long-term potentiation of glutamatergic synaptic transmission following a spike-timing-dependent protocol. Lastly, CIE treatment diminished the inhibitory effect of acute ethanol on AP spiking of IOFC neurons and reduced expression of the GlyTI transporter. Taken together, these results suggest that chronic exposure to ethanol leads to enhanced intrinsic excitability and glutamatergic synaptic signaling of IOFC neurons. These alterations may contribute to the impairment of OFC-dependent behaviors in alcohol-dependent individuals.
\end{abstract}

Neuropsychopharmacology (20 I6) 4I, I I I2-I I27; doi: I 0. I038/npp.20 I5.250; published online 9 September 20I5

\section{INTRODUCTION}

The orbitofrontal cortex (OFC) is a brain region within the prefrontal cortex (PFC) that reciprocally interacts with several brain areas, including the dorsal and ventral striatum, medial PFC (mPFC), amygdala, dorsal raphe nucleus, and midbrain (Kringelbach and Rolls, 2004). The OFC can be anatomically divided into medial and lateral regions, and has been suggested to have an important role in integrating sensory and reward-related information in support of several aspects of learning, including choice behavior (Bokura et al, 2001; Ramus and Eichenbaum, 2000; Roesch et al, 2007a; Schoenbaum et al, 1998), reversal learning (Bissonette et al, 2008; Dias et al, 1996; McAlonan and Brown, 2003; Roesch et al, 2007b; Thorpe et al, 1983), and the development of

* Correspondence: Dr IJ Woodward, Department of Neuroscience, Medical University of South Carolina, 67 President Street, IOP456N, Charleston, SC 29425, USA, Tel: + I 843792 5225, Fax: + I 843792 7353, E-mail: woodward@musc.edu

Received 4 April 2015; revised 14 August 2015; accepted 15 August 2015; accepted article preview online 19 August 2015 anticipation in response to either appetitive or aversive stimuli (Tremblay and Schultz, 1999). Lesions to the medial OFC result in the inability of animals to learn to associate a previously non-rewarded stimulus with reward, whereas damage to the lateral OFC (lOFC) impairs reversal learning that is needed to rapidly adjust responses when predicted outcomes change (Dias et al, 1996; Iversen and Mishkin, 1970; O'Doherty et al, 2001; Rogers et al, 1999b). Reversal learning deficits are also present in mice that display enhanced OFC firing due to genetic loss of parvalbumin interneurons (Bissonette et al, 2015), suggesting that dynamic changes in OFC activity are needed for optimal control of behavior.

Several studies have demonstrated an involvement of the OFC in drug addiction. The OFC, together with other limbic cortical areas, is stimulated when addicts are exposed to drug-associated stimuli that elicit intoxication, craving, and bingeing (Goldstein et al, 2007; Goldstein and Volkow, 2002). Chronic exposure to psychomotor stimulants including amphetamine or cocaine, and opiates produce changes in spine density and synaptic organization in the OFC 
(Robinson et al, 2001; Robinson and Kolb, 1999). Changes in OFC morphology are correlated with abnormalities in behaviors, especially the development and maintenance of social interactions (Kolb et al, 2004). In humans, neuroimaging studies reveal reduced gray matter density in the OFC of chronic cocaine users (Matochik et al, 2003). In addicts, dysfunction in decision-making and development of compulsive drug-seeking behaviors despite negative outcomes is similar to that observed in individuals with OFC damage (Bechara, 2005; Bechara and Van Der Linden, 2005; Rogers et al, 1999a). Impaired reversal learning has been found in animals following repeated intermittent cocaine treatment, indicating a malfunction of the 1OFC (Jentsch et al, 2002; Schoenbaum et al, 2004). In addition, persistent metabolic and neurochemical changes have been observed in the OFC of both drug addicts and drug abstinent subjects, suggesting that functional changes of the OFC may contribute to the development of drug addiction (Volkow et al, 2004).

The OFC has also been suggested to have an important role in the development of alcohol-abuse disorders. Acute exposure to ethanol disrupts reversal learning in monkeys (Jedema et al, 2011) and rats (Brown et al, 2007), and suppresses electrophysiological activity of lOFC neurons in mice (Badanich et al, 2013). Previous studies in our laboratory also demonstrate that mice exposed chronically to ethanol show impaired performance on a reversal learning task (Badanich et al, 2011) shown to require the lOFC (Bissonette et al, 2008). These findings are similar to those reported in human alcoholics (Fortier et al, 2008, 2009; Verdejo-Garcia et al, 2006), suggesting that alcohol-induced changes in OFC function may lead to loss of behavioral flexibility and poor judgment. Despite these intriguing findings, the effects of chronic alcohol exposure on the fundamental electrophysiological properties of OFC neurons are unknown. In the present study, we used whole-cell patchclamp electrophysiology and a well-validated mouse model of alcohol dependence, to examine how chronic exposure to ethanol affects deep-layer pyramidal neurons in the lateral OFC.

\section{MATERIALS AND METHODS}

\section{Animals}

Male C57/BL6J mice (9 weeks of age at beginning of study) were obtained from Jackson Laboratories (Bar Harbor, ME), and were group-housed (four/cage) and allowed to acclimatize to the colony room for at least 1 week in a temperatureand humidity-controlled AAALAC-approved facility, with ad libitum access to food and water. The animal colony room was maintained on a 12-h light/dark cycle with lights off at $0900 \mathrm{~h}$. All animals were treated in strict accordance with the NIH Guide for the Care and Use of Laboratory Animals, and all experimental methods were approved by MUSC's IACUC.

\section{Chronic Intermittent Ethanol Exposure}

Chronic intermittent ethanol (CIE) exposure of mice was initiated using a protocol previously shown to produce an impairment of OFC-dependent reversal learning (Badanich et al, 2011). Briefly, mice were exposed to four cycles of either ethanol (CIE group) or air (control) in vapor inhalation chambers. Each cycle consisted of daily exposure to ethanol vapor for $16 \mathrm{~h}$ followed by $8 \mathrm{~h}$ of abstinence in the home cage. This was repeated each day for 4 consecutive days, followed by 3 days of abstinence before beginning the next cycle of CIE exposure. Ethanol (95\%) was volatized by passing air through a submerged air stone and the resulting vapor was mixed with fresh air and delivered to Plexiglas inhalation chambers $(5 \mathrm{l} / \mathrm{min})$ to maintain consistent ethanol concentrations between 17 and $21 \mathrm{mg} / \mathrm{l}$ air in the chamber. This yielded blood ethanol concentrations (BECs) in the range of $150-250 \mathrm{mg} / \mathrm{dl}$. Before entry into the ethanol chambers, CIE mice were injected intraperitoneally $(20 \mathrm{ml} /$ $\mathrm{kg}$ body weight) with ethanol $(1.6 \mathrm{~g} / \mathrm{kg} ; 8 \% \mathrm{w} / \mathrm{v})$ and the alcohol dehydrogenase inhibitor pyrazole $(1 \mathrm{mmol} / \mathrm{kg})$, to maintain stable blood ethanol levels, whereas air control mice were injected with saline and pyrazole. Chamber ethanol concentrations were monitored daily and blood samples were collected weekly to monitor BECs during each CIE cycle. Mice were killed 3, 7 or 10 days following the final ethanol or air exposure for tissue collection.

\section{Preparation of Brain Slices}

Brain slices containing the 1OFC were prepared for wholecell patch-clamp electrophysiology experiments as previously described (Badanich et al, 2013). Following brief anesthesia with isoflurane and rapid removal of the brain, the tissue was blocked coronally for the frontal cortex; the cerebellum and a portion of the dorsal mesencephalon were removed. The tissue block was mounted in a Leica VT1000S vibratome (Buffalo Grove, IL) containing ice-cold oxygenated $\left(95 \% \mathrm{O}_{2}\right.$, $\left.5 \% \mathrm{CO}_{2}\right)$ sucrose containing buffer and coronal sections $(300 \mu \mathrm{m})$ were cut. Slices containing the lOFC were immediately placed in a holding chamber containing oxygenated artificial cerebral spinal fluid (aCSF) at $34^{\circ} \mathrm{C}$ for $30 \mathrm{~min}$ and kept at room temperature for at least $30 \mathrm{~min}$ before recordings. The composition of the cutting solution used was (in mM): 200 sucrose, $1.9 \mathrm{KCl}, 1.2 \mathrm{NaH}_{2} \mathrm{PO}_{4}$, $6 \mathrm{MgCl}_{2}, 0.5 \mathrm{CaCl}_{2}, 0.4$ ascorbate, 10 glucose, $25 \mathrm{NaHCO}_{3}$, adjusted to 305-315 mOsm. The composition of the aCSF was (in mM): $125 \mathrm{NaCl}, 2.5 \mathrm{KCl}, 1.25 \mathrm{NaH}_{2} \mathrm{PO}_{4}, 1.3 \mathrm{MgCl}_{2}$, $2.0 \mathrm{CaCl}_{2}, 0.4$ ascorbate, 10 glucose, $25 \mathrm{NaHCO}_{3}$, adjusted to 290-310 mOsm. Both solutions were saturated with $95 \% \mathrm{O}_{2} /$ $5 \% \mathrm{CO}_{2}(\mathrm{pH} 7.4)$.

\section{Whole-Cell Patch-Clamp Electrophysiology}

An individual slice was placed in the recording chamber and perfused with $34^{\circ} \mathrm{C}$ aCSF maintained at a flow rate of $2 \mathrm{ml} /$ min. Recordings were localized to deep layers of the lOFC using an Olympus BX51W1 microscope (Center Valley, PA) equipped with infrared Dodt gradient contrast imaging (Luigs and Neumann, Ratingen, Germany). Thin-wall borosilicate glass electrodes $(\mathrm{OD}=1.5 \mathrm{~mm}, \mathrm{ID}=1.17 \mathrm{~mm}$ ) were pulled on a Sutter Instrument P97 Micropipette Puller (Novato, CA) and had tip resistances ranging from 1.9 to $5.5 \mathrm{M} \Omega$. Patch pipettes filled with an internal solution were slowly lowered onto the layer $\mathrm{V}$ pyramidal neurons to obtain a seal ( $>1 \mathrm{GOhm}$ ) followed by breakthrough to gain wholecell access. All the whole-cell recordings were carried out using an Axon MultiClamp 700B amplifier (Molecular 
Devices, Union City, CA) and Instrutech ITC-18 analogdigital converter (HEKA Instruments, Bellmore, NY) controlled by AxographX software (Axograph, Sydney, Australia) running on a Macintosh G4 computer (Apple, Cupertino, CA). Events were filtered at $4 \mathrm{kHz}$ and digitized at a sampling rate of $10 \mathrm{kHz}$.

\section{Intrinsic Excitability Experiments}

To determine the effects of withdrawal (WD) from CIE on the intrinsic excitability of OFC neurons, current-clamp recordings were performed. Spike firing was induced by direct current injection $(750 \mathrm{~ms})$ through patch pipettes filled with a potassium gluconate internal solution (in $\mathrm{mM}$ : 120 K-gluconate, $10 \mathrm{KCl}, 10 \mathrm{HEPES}, 2 \mathrm{MgCl}_{2}, 1 \mathrm{EGTA}, 2$ NaATP, $0.3 \mathrm{NaGTP}$, adjusted to $294 \mathrm{mOsm}, \mathrm{pH}$ 7.4). Recordings were analyzed for the number of spikes in response to each current step, resting membrane potential (RMP, $\mathrm{mV})$, height $(\mathrm{mV})$, half width (ms), rise time (ms), and after-hyperpolarization (AHP, $\mathrm{mV}$ ). RMP was obtained from the membrane potential just before initiation of current steps. AHP magnitude was calculated by subtracting the lowest potential during hyperpolarization from action potential (AP) threshold and reported values are the mean of the first three AHP magnitudes recorded. The magnitudes of the depolarizing current pulse used to quantify the AHPs ranged from 40 to $80 \mathrm{pA}$.

\section{SK Tail Current}

To directly determine small conductance calcium-activated potassium (SK) currents from lOFC neurons, a potassium methylsulfate internal pipette solution (in $\mathrm{mM}: 140$ K-methylsulfate, $8.0 \mathrm{NaCl}, 1.0 \mathrm{MgCl}_{2}, 0.05$ EGTA, 10 HEPES, and $5 \mathrm{Mg}_{2} \mathrm{ATP}$, adjusted to $275-285 \mathrm{mOsm}, \mathrm{pH}$ 7.4) was used. A low concentration of calcium chelator EGTA was included in the pipette solution to preserve calciumdependent potassium currents during voltage-clamp recordings. Neurons were voltage-clamped at $-70 \mathrm{mV}$ and depolarizing current steps $(400 \mathrm{~ms}$, from -20 to $+30 \mathrm{mV}$ with $10 \mathrm{mV}$ between steps) were applied before being brought back to $-70 \mathrm{mV}$. Tail currents were measured again in the presence of the SK channel inhibitor apamin $(100 \mathrm{nM})$ that was locally applied using the gravity-fed perfusion barrel positioned just above the recording area as described previously (Badanich et al, 2013). Specifically, separate glass syringes were used as perfusion reservoirs and a stopcock controlled the flow of each drug solution into a four-way manifold connected to a square quartz perfusion tube (0.6 mm ID; Warner Instruments, Hamden, CT). SK currents were defined as the difference in peak magnitudes of tail currents obtained in the absence and the presence of apamin.

\section{Evoked and Spontaneous Synaptic Currents}

A cesium chloride internal pipette solution (in mM: 120 CsCl, 10 HEPES, $2 \mathrm{MgCl}_{2}$, 1 EGTA, 2 NaATP, $0.3 \mathrm{NaGTP}, 1$ QX-314, adjusted to $294 \mathrm{mOsm}, \mathrm{pH}$ 7.4) was used to record synaptic NMDA- and AMPA-mediated excitatory postsynaptic currents (EPSCs) in voltage-clamp mode. A concentric bipolar stimulating microelectrode (World Precision Instruments, Sarasota, FL) was placed onto the lOFC adjacent to and in close proximity to the recording site. Stimulus pulses were delivered at a rate of $0.01 \mathrm{~Hz}$ and stimulation strength was adjusted $(10-1025 \mu \mathrm{A})$, to elicit a stable and submaximal evoked current. EPSCs were evoked at a holding potential of $+40 \mathrm{mV}$ in the presence of picrotoxin (100 $\mu \mathrm{M}$; AMPA+NMDA EPSC) and then picrotoxin plus AP5 $(100 \mu \mathrm{M})$ was washed in to isolate the AMPA component. The average AMPA EPSC was digitally subtracted from the total EPSC to yield the NMDA component and the AMPA/NMDA ratio was calculated for each neuron. Amplitude (pA) and area (pA/ms) of synaptic events were measured to determine the effects of WD from CIE on evoked AMPA and NMDA EPSCs. In separate set of experiments, a cesium methanesulfonate internal pipette solution (in mM: $125 \mathrm{CsMeSO}_{3}, 10 \mathrm{CsCl}, 5 \mathrm{NaCl}, 10$ HEPES, 1 EGTA, $2 \mathrm{MgCl}_{2}, 5 \mathrm{MgATP}, 0.3 \mathrm{NaGTP}$ ) was used to measure spontaneous EPSCs (sEPSCs) and inhibitory postsynaptic currents (IPSCs) in the same neuron by recording events at a membrane potential at -70 or $10 \mathrm{mV}$, respectively (Ma et al, 2013). Spontaneous events were detected using a template matching algorithm and a threshold amplitude of $6 \mathrm{pA}$ for sEPSCs and $10 \mathrm{pA}$ for spontaneous IPSCs (sIPSCs). Miniature EPSCs (mEPSCs) were measured using a $\mathrm{CsCl}$ containing internal and aCSF containing tetrodotoxin (TTX; $1 \mu \mathrm{M}$ ) and picrotoxin. Events were detected offline in AxographX software using a template-matching algorithm.

\section{Spike-Timing-Dependent Plasticity}

Long-term potentiation (LTP) of glutamatergic synaptic transmission was examined using a spike-timing-dependent plasticity (STDP) protocol as described previously (Kroener et al, 2012). With a potassium gluconate internal solution, EPSPs were evoked at $0.1 \mathrm{~Hz}$ at $-70 \mathrm{mV}$ until a stable baseline was obtained for $10 \mathrm{~min}$. STDP was then induced by 48 pairings of evoked EPSPs and postsynaptic APs at $0.1 \mathrm{~Hz}$. Postsynaptic APs were evoked by brief $(2 \mathrm{~ms} ; 2 \mathrm{nA})$ somatic current injections consisting of 5 pulses $(200 \mathrm{~Hz})$. Stimulation of the EPSP preceded the middle AP of the burst by $2 \mathrm{~ms}$. Mean changes of EPSP amplitudes before STDP were compared with mean changes of EPSP amplitudes 20-30 min and 50-60 min after STDP induction.

\section{Tonic Currents}

Changes in tonic current after WD from CIE in the absence or presence of receptor blockers or acute ethanol were measured by recording the holding current of a neuron voltage clamped at $-70 \mathrm{mV}$. In these experiments, pipettes were filled with a cesium chloride internal solution. Receptor blockers or ethanol were locally applied to the patched neuron using a gravity-fed perfusion barrel described above. Before administration, holding current was measured during local perfusion of aCSF to obtain a stable baseline (2-3 min). Then, the solution was switched to one containing the $\mathrm{GABA}_{\mathrm{A}}$ receptor blocker gabazine $(10 \mu \mathrm{M})$ for $5 \mathrm{~min}$, followed by a combination of gabazine $(10 \mu \mathrm{M})$ plus the glycine receptor blocker strychnine $(1 \mu \mathrm{M})$. In a separate group of neurons, the change in holding current produced by local application of ethanol $(33-66 \mathrm{mM})$ was measured. Tonic currents were analyzed offline and were measured as 
changes in current relative to baseline (eg, $I_{\mathrm{Gabazine}}-I_{\mathrm{aCSF}}$ and $I_{\text {Gabazine/Strychnine }}-I_{\text {Gabazine }}$ ).

\section{Drugs}

Picrotoxin, AP5, gabazine, TTX citrate, sarcosine, kynurenic acid, CGP55845, and apamin were purchased from Tocris Biosciences (Minneapolis, MN). Ethanol was purchased from Pharmco-AAPER (Brookfield, CT). Strychnine and all reagents used to prepare aCSF, sucrose-containing and internal pipette solutions were purchased from Sigma (St Louis, MO).

\section{Western Blotting}

Subcellular fractionation and western blotting were performed as described previously (McGuier et al, 2015). Briefly, tissue punches were prepared from the 1OFC of control and CIE-treated mice at 3 or 7 days following the last exposure. A Dounce homogenate was prepared and centrifuged at $23000 \mathrm{~g}$ for $30 \mathrm{~min}$ at $4{ }^{\circ} \mathrm{C}$. The pellet containing membrane-associated proteins was resuspended with $0.5 \%$ Triton X-100 buffer. The suspension was rotated for $15 \mathrm{~min}$ at $4{ }^{\circ} \mathrm{C}$ and then centrifuged at $12000 \mathrm{~g}$ for $20 \mathrm{~min}$ at $4{ }^{\circ} \mathrm{C}$, generating detergent-soluble and -insoluble fractions. The detergent-insoluble pellet was solubilized in 2\% LDS and western blottings were performed on this fraction. An aliquot of each sample was taken for determination of protein concentration by the bicinchoninic acid assay (Pierce Biotechnology, Rockford, IL). The remaining samples were stored at $-80^{\circ} \mathrm{C}$ until immunoblot analysis. Samples were diluted with NuPAGE $4 \times$ LDS sample loading buffer (Invitrogen, Carlsbad, CA; pH 8.5) containing $50 \mathrm{mM}$ dithiothreitol and samples were denatured for $10 \mathrm{~min}$ at $70^{\circ} \mathrm{C}$. Ten micrograms of each sample was separated using the Bis-Tris $(375 \mathrm{mM}$ resolving buffer and $125 \mathrm{mM}$ stacking buffer, $\mathrm{pH}$ 6.4; 7.5\% acrylamide) discontinuous buffer system with MOPS electrophoresis buffer (50 mM MOPS, $50 \mathrm{mM}$ Tris, $0.1 \%$ SDS, $1 \mathrm{mM}$ EDTA, pH 7.7). Protein was then transferred to Immobilon-P PVDF membranes (Millipore, Bedford, MA) using a semi-dry transfer apparatus (Bio-Rad Laboratories, Hercules, CA). After transfer, blots were washed with phosphate-buffered saline containing $0.1 \%$ Tween 20 (PBST) and then blocked with PBST containing $5 \%$ nonfat dried milk (NFDM) for $1 \mathrm{~h}$ at room temperature with agitation. The membranes were then incubated overnight at $4{ }^{\circ} \mathrm{C}$ with primary antibodies diluted in PBST containing $0.5 \%$ NFDM and washed in PBST before $1 \mathrm{~h}$ incubation at room temperature, with horseradish peroxidase-conjugated secondary antibodies diluted 1:2000 in PBST. Membranes received a final wash in PBST and the antigen-antibody complex was detected by enhanced chemiluminescence using a ChemiDoc MP Imaging system (Bio-Rad Laboratories). The bands were quantified by mean optical density using computer-assisted densitometry with ImageJ v1.41 (National Institutes of Health, USA). As loading controls (eg, actin and GAPDH) often used for in western blot experiments are subject to quantitation errors (Aldridge et al, 2008; Dittmer and Dittmer, 2006), each gel was normalized to a total protein stain (Swift stain; G-Biosciences, St Louis, MO). Before each study, a series of western blottings were performed using different titrations of sample and antibody, to establish the linear range for each response. Antibodies against the various proteins were obtained from the following sources and used at the indicated dilution. GluN1 (BD Pharmingen, San Jose, CA, catalog number 556308; 1:000), GluN2A (EMD Millipore, Billerica, MA, catalog number 07-732; 1:2000), GluN2B (NeuroMab, Davis, CA, catalog number 75-097; 1:2000), GluA1 (EMD Millipore, catalog number AB1504; 1:1000), GluA2 (Neuromab, catalog number 75-002; 1:1000), SK2 (Frontier Institute, Hokkaido, Japan, catalog number SK2Rb-Af500; 1:1000), SK3 (Alomone Labs, Jerusalem, Israel, catalog number APC-025; 1:2000), and glycine receptor (Synaptic Systems, Germany, catalog number mAb4, 1:2000). The GlyT1 antibody was kindly provided by Dr Francisco Zafra, Universidad Autónoma, Madrid, Spain and was used at a dilution of 1:1000.

\section{Statistical Analysis}

Experimental data are expressed as the mean \pm SEM and were analyzed with Prism software (GraphPad Software, San Diego, CA) using standard methods (ANOVA, $t$-test and so on) as indicated. Comparisons were considered significantly different when $p<0.05$.

\section{RESULTS}

Mice were treated with 4 weekly cycles (4 days per week, $16 \mathrm{~h}$ per day) of ethanol exposure using vapor chambers. The average BEC during the 4 weeks of CIE exposure was $219.5 \pm 5.9 \mathrm{mg} / \mathrm{dl}$. Following the last treatment, CIE mice were returned to the animal colony room and used for electrophysiological recordings at 3, 7, or 10 days post WD. The previous study from our laboratory (Badanich et al, 2013) reported two types of IOFC neurons: large, regularspiking cells with input resistance $<100 \mathrm{M} \Omega$ and a small subset of small, fast-spiking neurons with higher input resistance and a deep AHP following each AP. All patchclamp experiments in the present study were obtained from large, regular-spiking lOFC neurons.

\section{WD from CIE Enhances the Excitability of 10FC Neurons}

A potassium gluconate internal pipette solution was used to determine the effects of CIE exposure on the intrinsic excitability of IOFC neurons. Under current-clamp mode, APs were induced by direct current injection (from 40 to $160 \mathrm{pA}$, with $20 \mathrm{pA}$ increments). Representative traces demonstrating current-induced spike firing under each condition (air treated, 3-day WD, 7-day WD, and 10-day WD) are shown in Figure 1a. As summarized in Figure 1b, CIE exposure produced a robust increase in AP number in lOFC neurons as compared with air controls (two-way ANOVA: main effect of CIE, $\left.\mathrm{F}_{(3,308)}=27.96, p<0.0001\right)$. Post-hoc comparisons showed that across all current steps, this effect was highly significant for both the 3-day WD group $(q=8.03, p<0.0001)$ and the 7 -day WD group $(q=6.93, p<0.0001)$. As compared with controls, spiking over all current steps was also elevated in the 10-day WD group, although to a lesser extent $(q=2.53, p=0.036)$. The increase in AP number in CIE groups was associated with a significant reduction in the amplitude of the AHP (Figure 1c, main effect of CIE $\left.\mathrm{F}_{(3,75)}=6.703, p=0.0005\right)$. Post-hoc 
a

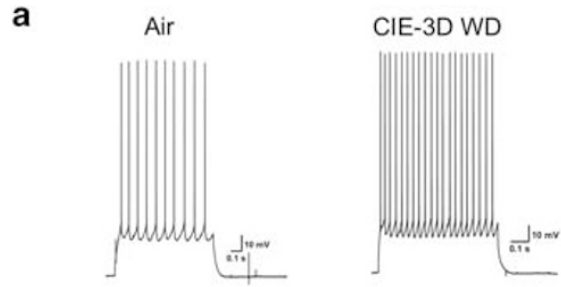

b

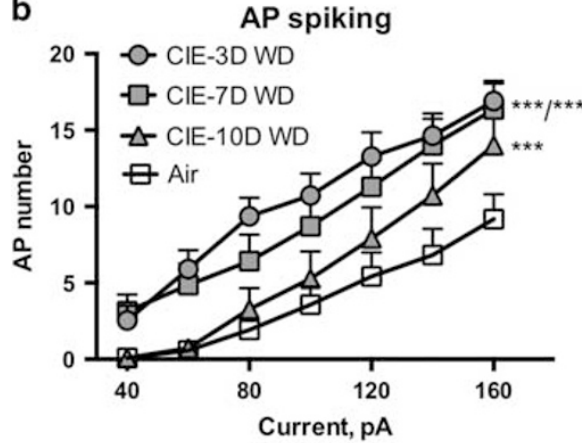

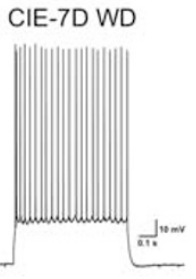

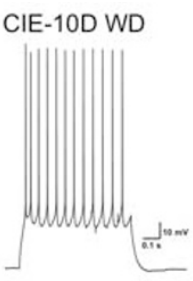

C

After-hyperpolarization

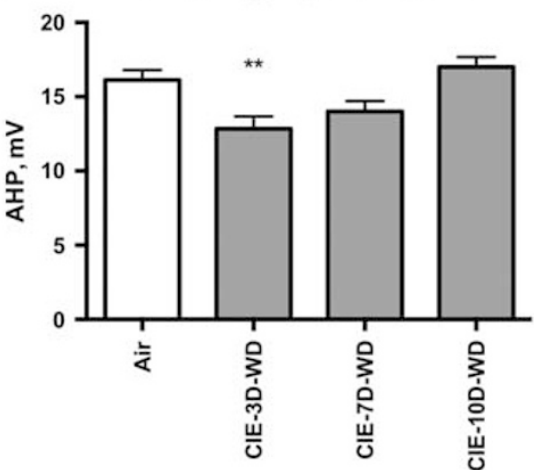

Figure I Chronic intermittent ethanol (CIE) exposure enhances current-induced spiking in lateral orbitofrontal cortex (IOFC) neurons and decreases the magnitude of the after-hyperpolarization (AHP). (a) Representative traces showing enhanced action potential spiking in all CIE-exposed groups as compared with air-treated controls. (b) Number (mean \pm SEM) of spikes from IOFC neurons plotted against a series of current injections (40-160 pA). In comparison with air control $(n=12)$, basal firing rates of IOFC neurons were significantly enhanced in 3-day withdrawal (3D-WD; $n=11)$, 7-day WD (7D-WD; $n=\mid 4)$, and I0-day WD (IOD-WD; $n=1$ I) ClE groups (two-way analysis of variance (ANOVA): main effect of $\mathrm{ClE}_{\text {, }} \mathrm{F}_{(3,308)}=27.96, p<0.000$ I; post-hoc comparison; air vs $\mathrm{ClE}$ treated; **** $<0.000 \mathrm{I}$ ). (c) Effects of CIE treatment on AHP. CIE induced a significant reduction in AHP amplitude (two-way ANOVA: main effect of $\mathrm{CIE}, \mathrm{F}_{(3,75)}=6.70, p<0.0005$; post-hoc comparison, air vs $\mathrm{ClE}$ treated, $* *+0.0 \mathrm{I}$ ) at 3D-WD, but not at the 7-day and I0-day time points.

Table I Electrophysiological Characteristics of OFC Neurons

\begin{tabular}{|c|c|c|c|c|c|c|}
\hline Treatment $(N)$ & RMP $(\mathrm{mV})$ & IR $(\mathbf{M} \Omega)$ & AP threshold $(\mathrm{mV})$ & AP height $(\mathrm{mV})$ & AP width (ms) & AP rise (ms) \\
\hline Air (12) & $-67.9 \pm 2.4$ & $61.5 \pm 3.2$ & $-40.1 \pm 1.8$ & $55.2 \pm 3.1$ & $0.99 \pm 0.17$ & $0.41 \pm 0.04$ \\
\hline 3D-WD (II) & $-65.0 \pm 2.3$ & $63.9 \pm 4.1$ & $-41.9 \pm 2.1$ & $57.0 \pm 7.0$ & $0.75 \pm 0.04$ & $0.36 \pm 0.04$ \\
\hline 7D-WD (I4) & $-71.5 \pm 2.7$ & $63.8 \pm 2.6$ & $-41.3 \pm 2.5$ & $55.0 \pm 3.3$ & $0.82 \pm 0.76$ & $0.37 \pm 0.05$ \\
\hline IOD-WD (II) & $-70.8 \pm 0.5$ & $68.7 \pm 4.1$ & $-38.6 \pm 0.7$ & $52.6 \pm 3.2$ & $0.82 \pm 0.03$ & $0.42 \pm 0.06$ \\
\hline
\end{tabular}

Abbreviations: AP, action potential; IR, input resistance; RMP, resting membrane potential.

Values are mean $( \pm$ SEM).

comparisons revealed that the AHP in the 3-day WD group was significantly less than the air control $(q=3.095$, $p=0.0077$ ), whereas that for the 7-day and 10-day CIE groups were not different. Other electrophysiological characteristics of lOFC neurons obtained from air and CIE mice are summarized in Table 1 . There were no significant differences in the RMP, input resistance, AP threshold, AP height, AP width, or AP rise between the groups (all one-way ANOVAs: $p>0.05$ ).

The increase in neuronal excitability in IOFC neurons was not due to altered synaptic activity, as similar changes were found when recordings were performed in the combined presence of blockers of synaptic glutamate (kynurenic acid, $1 \mathrm{mM}$ ), GABA $\mathrm{A} / \mathrm{B}$ (picrotoxin, $100 \mu \mathrm{M}$; CGP55845, 1 $\mu \mathrm{M}$ ), and glycine receptors (strychnine, $1 \mu \mathrm{M}$; Supplementary Figure S1).

As internal solution can affect a stability of whole-cell recording by altering the internal milieu of the neuron
(Kaczorowski et al, 2007; Kay, 1992), we also examined the effect of K-gluconate internal solution on current-induced spiking and AHPs in a subset of control neurons $(n=4)$ every $15 \mathrm{~min}$ for a 45 -min recording period (Supplementary Figure S2). With K-gluconate as the internal, there were no significant differences in AP firing over the time course (twoway ANOVA: $\left.F_{(3,9)}=2.619, p=0.115\right)$. Mean $( \pm$ SEM) AHP amplitude at $0,15,30$, and $45 \mathrm{~min}$ were also not different (one-way ANOVA: $\mathrm{F}_{(3,12)}=0.103, p=0.9567$ ), suggesting that K-gluconate does not affect intrinsic neuronal excitability of $1 \mathrm{OFC}$ neurons over a long recording period.

\section{WD from CIE Suppresses the Functional Activity of Small Conductance SK Channels}

The decrease in the amplitude of AHP in CIE-treated mice may have contributed to the increase in AP firing in neurons from these animals. AHPs are divided into fast, medium, and 
a

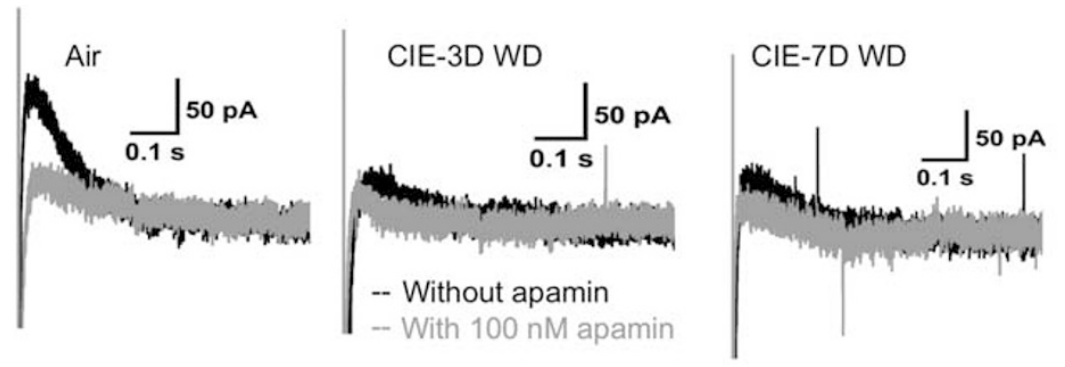

b

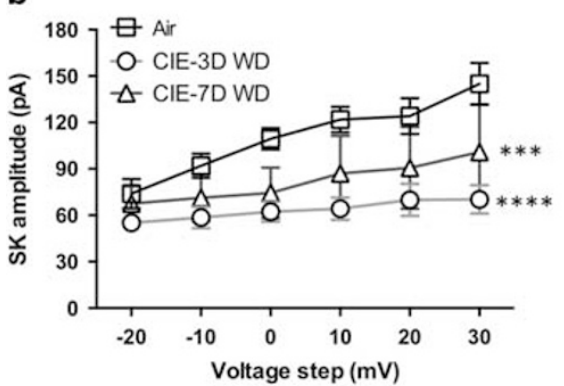

C
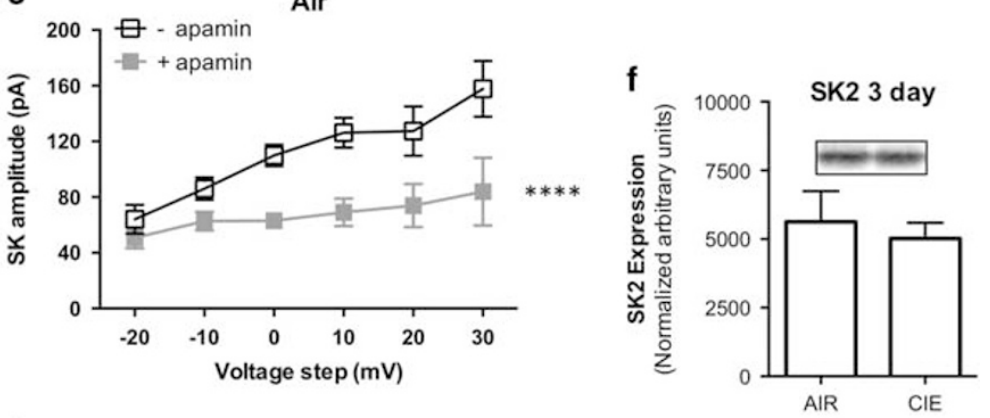

d

CIE-3D WD

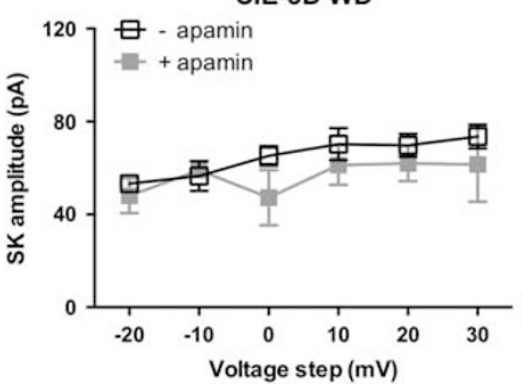

e

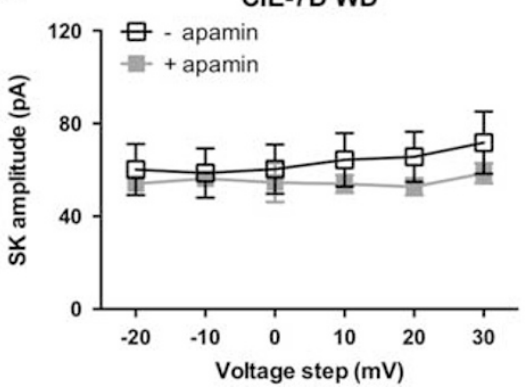

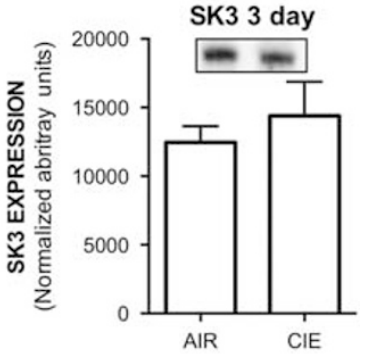

Figure 2 Chronic intermittent ethanol (CIE) exposure reduces the amplitude of apamin-sensitive tail currents without changing small conductance calciumactivated potassium (SK) channel subunit expression. (a) Representative traces showing a decrease in total tail current amplitudes in CIE-exposed mice. In addition, shown are traces in the presence of the SK channel blocker apamin showing reduction in SK currents in CIE-exposed animals. (b) Comparison of the peak tail current amplitudes (mean \pm SEM) in response to depolarizing voltage steps ( $400 \mathrm{~ms}$, from -20 to $+30 \mathrm{mV}$ with $10 \mathrm{mV}$ between steps). There was a significant main effect of CIE treatment on total tail current amplitude (two-way analysis of variance (ANOVA): $\left.F_{(2,150)}=34.76, p<0.000 \mathrm{I}\right)$ with amplitudes from both the 3-day withdrawal (WD; $q=8.3 \mathrm{I}$, ******* $p<0.000 \mathrm{I} ; n=12)$ and 7 -day WD $(q=3.93$; $* * * * 0.00 \mathrm{I} ; n=5)$ ClE groups being significantly different from the control $(n=\mid I)$. The SK channel blocker apamin $(I 00 \mathrm{nM})$ significantly reduced tail current amplitudes detected in lateral orbitofrontal cortex (IOFC) neurons from air control mice (c; two-way ANOVA: $F_{(I, 72)}=33.76$, ***** $p<0.000 \mathrm{I} ; n=7$ ) but did not significantly change the tail current amplitude in 3 -day (d; two-way ANOVA: $F_{(I, 36)}=3.217, p=0.0813 ; n=4$ ) and 7-day (e; two-way ANOVA: $\left.F_{(I, 36)}=2.845, p=0.1003 ; n=4\right)$ WD groups. (f) Representative western blottings and optical density analysis of SK2 and SK3 subunits in IOFC neurons from air- and ClE-exposed mice. In comparison with air controls, there were no significant changes in SK2 or SK3 protein expression in CIE-treated mice at the 3 -day WD time point $(S K 2$-test, $t(9)=0.5 \mathrm{I}, p=0.62 ;$ SK3 $t$-test, $t(20)=0.70, p=0.49)$.

slow components, with approximate durations of $2-5 \mathrm{~ms}$, 50-200 ms, and >0.5 s, respectively (Sah, 1996; Sah and Faber, 2002). The duration of the AHP obtained from lOFC neurons in this study ranged from 64.2 to $188.44 \mathrm{~ms}$ with the average of $109 \pm 4.99 \mathrm{~ms}(n=46)$, consistent with an mAHP. Ion channels that underlie the mAHP include apaminsensitive SK channels (Villalobos et al, 2004) and decreases in SK channel expression or function are known to drive increased AP firing (Brodie and Appel, 1998; Brodie et al, 1999; Lopez de Armentia and Sah, 2004). To investigate the effects of CIE treatment on SK channel function, depolarizing voltage steps $(400 \mathrm{~ms}$, from -20 to $+30 \mathrm{mV}$ with $10 \mathrm{mV}$ between steps) were applied to lOFC neurons to activate an outward tail current that is mediated in part by SK channels. Figure 2a shows representative traces of tail currents from air- and ethanol-exposed mice obtained in the absence and presence of apamin. There was a significant main effect of CIE treatment on total tail current amplitude (two-way ANOVA: $\mathrm{F}_{(2,150)}=34.76, p<0.0001$; Figure $2 \mathrm{~b}$ ) with amplitudes from both the 3-day $(q=8.31, p<0.0001)$ and 7 -day $(q=3.93 ; p<0.001)$ CIE groups being significantly different from the control. In air control mice, apamin (100 nM) significantly suppressed tail currents (two-way ANOVA: $\mathrm{F}_{(1,96)}=43.18, p<0.0001$, Figure $\left.2 \mathrm{c}\right)$, indicating the involvement of SK channels in these responses. In contrast, apamin had no significant effect on tail currents in neurons from CIE-exposed mice withdrawn from ethanol for 3 days (twoway ANOVA: $F_{(1,84)}=1.329, p=0.2523$, Figure $2 d$ ) or 7 days (two-way ANOVA: $\mathrm{F}_{(5,36)}=0.126, p=0.99$, Figure $2 \mathrm{e}$ ). It is noteworthy that in these studies, a potassium methylsulfate internal pipette solution was used, as previous reports suggest that in some neurons, SK channels may be affected 

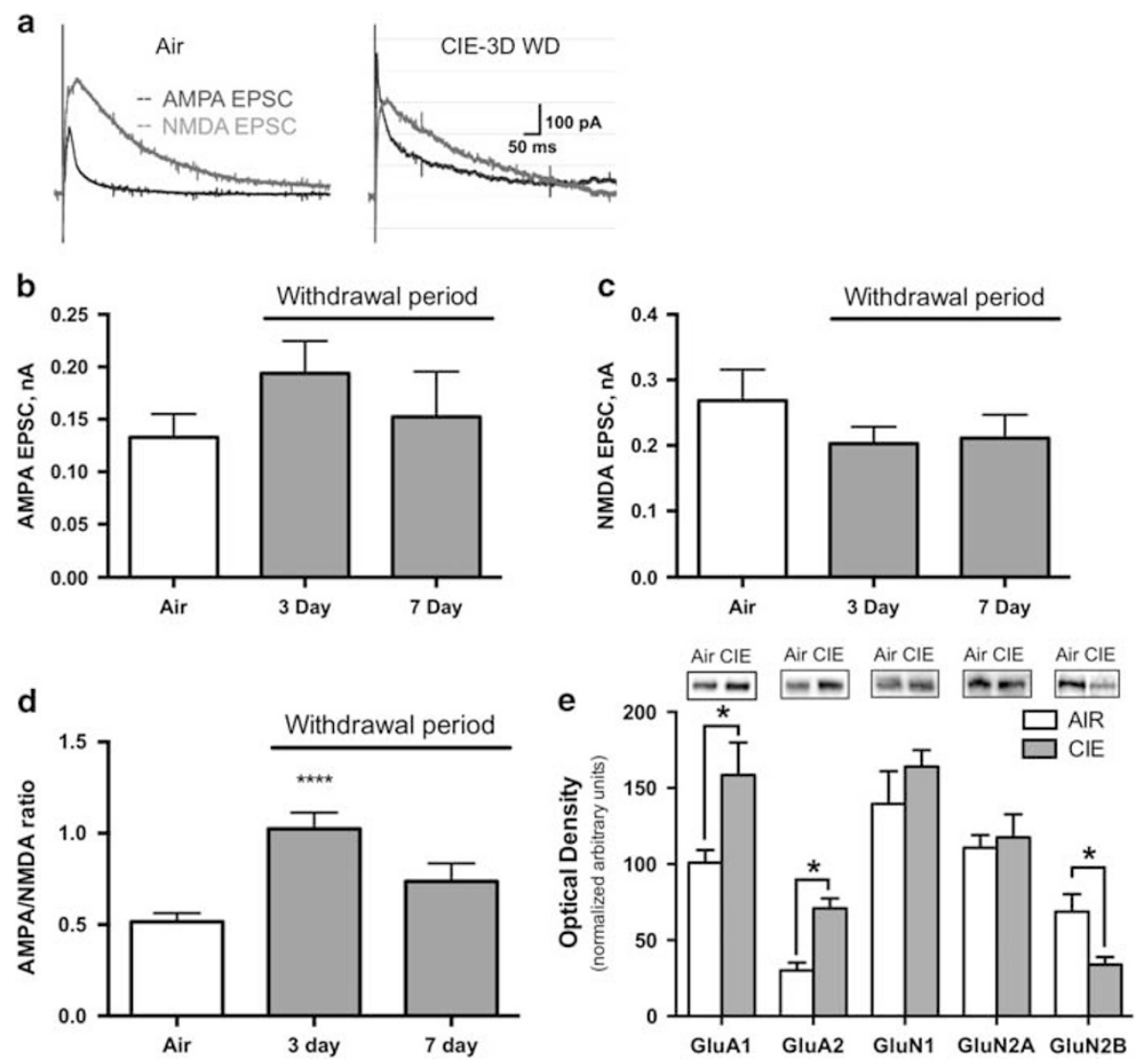

Figure 3 Chronic intermittent ethanol (CIE) exposure enhances the AMPA/NMDA excitatory postsynaptic current (EPSC) ratio and alters glutamatergic receptor subunit expression. (a) Representative traces demonstrating stimulus-evoked AMPA and NMDA EPSCs in lateral orbitofrontal cortex (IOFC) neurons from air- and CIE-exposed mice. CIE exposure produced a trend toward an increase in stimulus-evoked AMPA EPSCs (b; mean \pm SEM) and a decrease in stimulus-evoked NMDA EPSCs (c), but these changes were not statistically different from the control. (d) In comparison with air control mice $(n=16)$, CIE exposure produced a significant increase in AMPA/NMDA ratio at the 3-day withdrawal (WD) time point (one-way analysis of variance (ANOVA): $F_{(2,38)}=12.73$, ******* $p<0.000 \mathrm{I} ; n=14$ ). (e) Representative western blottings and optical density (mean \pm SEM) of AMPA and NMDA receptor subunits in IOFC neurons from air $(n=6)$ and 3-day WD $(n=5)$ groups. A significant increase in AMPA GluAI $(t(9)=2.69, * p=0.03)$ and GluA2 $(t(8)=5.00, * p=0.00 \mathrm{I})$ subunit expression was observed in CIE-exposed mice. CIE induced a significant decrease in NMDA GluN2B subunit expression $(t(8)=2.34, * 0.047)$. There were no differences in expression of NMDA GluNI and GluN2A subunits between control and CIE-exposed groups.

by the calcium chelator EGTA or gluconate (Velumian et al, 1997; Zhang et al, 1994). To determine whether this occurs in lOFC neurons, we compared the effects of different internal pipette solutions on SK currents in a small set of lOFC neurons from naive mice. Mean $( \pm$ SEM) tail amplitudes evoked at the highest depolarizing step $(+30 \mathrm{mV})$ were $176.46 \pm 16.86, \quad 205.47 \pm 56.78$, and $222.4 \pm 62.48 \mathrm{pA}$ for potassium gluconate with $1 \mathrm{mM}$ EGTA, potassium gluconate with no EGTA, and potassium methylsulfate, respectively. These values were not different from one another (two-way ANOVA: $\left.\mathrm{F}_{(2,60)}=0.688, p=0.507\right)$, suggesting that in lOFC neurons, gluconate containing internal solutions do not affect SK currents at least during the time of recording $(<5 \mathrm{~min})$.

Previous studies have reported the expression of three distinct subunits of SK channels throughout the brain (Sailer et al, 2002, 2004). In the cerebral cortex, SK1 is highly expressed in layer I to V with minimal expression in layer VI. SK2 mainly localizes in layer $\mathrm{V}$ and is sparsely found in layer II to IV. SK3 is moderately expressed in all layers of the cerebral cortex. Although SK1 subunits are abundantly expressed, only SK2 and SK3 channels are sensitive to apamin and these channels are thought to have a key role in contributing to the mAHP. In addition, chronic exposure to alcohol has been linked to decreases in SK2 and SK3 channel subunits in the human basolateral amygdala (Ponomarev et al, 2012) and rodent nucleus accumbens (Padula et al, 2015), respectively. To determine whether the decrease in SK tail currents in CIE-treated mice reflects a change in expression of SK channel subunits, western blotting with antibodies against SK2 and SK3 subunits was performed using tissue isolated from CIE-treated mice. As shown in Figure 2f, there was no significant change in SK2 or SK3 protein expression in the OFC in the 3-day CIE group as compared with air controls (SK2 $t$-test, $t(9)=0.51, p=0.62$; SK3 $t$-test, $t(20)=0.70, p=0.49$ ). There was also no change in SK2 expression noted at the 7-day CIE WD period (normalized arbitrary units; Control $72.96 \pm 4.34$; CIE $81.87 \pm 7.47 ;$-test, $t(12)=1.03, p=0.32)$.

\section{WD from CIE Enhances AMPA/NMDA EPSCs and Alters Receptor Subunit Expression}

We next examined whether WD from CIE treatment alters glutamatergic synaptic transmission of lOFC neurons by recording in each neuron stimulus-evoked AMPA and 
NMDA EPSCs. Representative traces demonstrating AMPA/ NMDA EPSC from air and CIE groups are shown in Figure 3a. CIE treatment produced a trend towards an increase in the amplitude of evoked AMPA EPSCs in 3-day WD mice (Figure $3 b$ ) with a corresponding reduction in NMDA-mediated EPSCs at both 3 days and 7 days post WD (Figure 3c). However, these changes were not statistically different from those obtained from air control mice, likely owing to the inherent variability in the amplitude of evoked glutamatergic EPSCs recorded from different neurons. CIE exposure did produce a robust increase in the AMPA/ NMDA ratio that was calculated for each neuron (one-way ANOVA: $F_{(2,38)}=12.73, p<0.0001$, Figure $3 \mathrm{~d}$ ) and pairwise comparisons revealed a significant increase in the ratio at the 3 -day time point $(q=5.037, p<0.0001)$. Western blotting for ionotropic glutamate receptors confirmed these findings and showed that 3-day CIE mice had a significant increase in the expression of GluA1 $(t(9)=2.69, p=0.03)$ and GluA2 $(t(8)=5.00, p=0.001)$ AMPA subunits and a corresponding decrease in the GluN2B NMDA subunit $(t(8)=2.34$, $p=0.047$ ) (Figure $3 \mathrm{e}$ ). There were no differences in expression of GluN1 or GluN2A NMDA subunits between air control and CIE-treated mice.

\section{WD from CIE Alters Spontaneous Synaptic Transmission of 10FC Neurons}

To further examine how CIE treatment affects synaptic transmission, we measured sEPSCs and sIPSCs. In these experiments, we used a modified internal pipette solution and two different holding potentials to monitor sEPSCs and sIPSCs in the same neuron ( $\mathrm{Ma}$ et al, 2013). As compared with air controls, CIE-treated mice showed increases in the amplitude (one-way ANOVA: $\mathrm{F}_{(3,39)}=2.889, p=0.0476$; Figure $4 \mathrm{a}$ ), but not frequency (Figure $4 \mathrm{~b}$ ) of spontaneous excitatory synaptic currents (measured as inward currents at $-70 \mathrm{mV}$ ). Pairwise comparisons revealed that sEPSC amplitude was significantly greater than controls at 3 days $(q=2.46, p<0.05)$ and 10 days $(q=2.55, p<0.05)$ of WD from CIE exposure. Increases in the amplitude but not frequency of sIPSCs (recorded at $+10 \mathrm{mV}$ ) were also observed in CIE-treated mice (one-way ANOVA: $\left.\mathrm{F}_{(3,39)}=6.205, p<0.01\right)$ and these were statistically different from air controls in 7-day $(q=3.74, p<0.01)$ and 10-day $(q=3.299, p<0.01)$ WD animals (Figure $3 c)$. A small set of follow-up studies on mEPSCs was performed using TTX $(1 \mu \mathrm{M})$ in the aCSF to block APs. Under these conditions, there was a trend towards an increase the amplitude of mEPSCs for CIE mice (Figure 4e; one-way ANOVA: main effect of CIE, $\mathrm{F}_{(2,18)}=2.858, p=0.08$ ), but pairwise comparisons revealed no statistically significant differences between control and CIE-treated mice. Similar to sEPSCs, CIE treatment had no effect on the frequency of AMPA mEPSCs (Figure 4f).

\section{WD from CIE Produces a Persistent Increase in Synaptic Plasticity Induced by STDP Protocol}

Increases in the AMPA/NMDA ratio and amplitude of EPSCs are usually interpreted as a sign of enhanced glutamatergic synaptic transmission. However, these measures provide only a static measure of synaptic efficacy and do not indicate whether the affected neurons show altered sensitivity to plasticity-inducing inputs. To determine whether CIE exposure produces dynamic changes in glutamatergic plasticity, we used a STDP protocol previously shown to produce a short-term potentiation of AMPAmediated EPSPs in the mPFC (Kroener et al, 2012). In these studies, single EPSPs were induced by focal electrical stimulation applied close to the recorded neuron. After a stable baseline of responses was collected, stimulationevoked EPSPs were paired with post-synaptic APs induced by brief current injections delivered through the pipette. Following the pairing protocol, single EPSPs were monitored and changes in EPSP amplitude were averaged over two 10 -min periods at $20-30$ and $50-60$ min post pairing. Mean $( \pm$ SEM) EPSP amplitudes of air-control group before and at 20-30 and 50-60 min post pairing were $3.41 \pm 0.17$, $5.15 \pm 0.2$, and $3.11 \pm 0.08 \mathrm{mV}$, respectively. Mean $( \pm$ SEM) EPSP amplitudes of CIE-3D WD group before and at 20-30 and 50-60 min post pairing were $3.49 \pm 0.05,5.51 \pm 0.09$, and $5.36 \pm 0.13 \mathrm{mV}$, respectively. Mean ( \pm SEM) EPSP amplitudes of CIE-7D WD group before and at 20-30 and $50-60 \mathrm{~min}$ post pairing were $4.19 \pm 0.12,5.97 \pm 0.08$, and $6.31 \pm 0.1 \mathrm{mV}$, respectively (Figure 5a). As shown in Figure 5b, the STDP protocol produced significant increases in EPSP amplitude that were time and CIE dependent (two-way ANOVA: main effect of time, $\mathrm{F}_{(1,48)}=5.576$, $p=0.0223$; main effect of CIE, $\mathrm{F}_{(2,48)}=6.808, p=0.0025$; time $\times$ CIE interaction, $\left.F_{(2,48)}=7.310, p=0.0017\right)$. Withingroup comparisons showed that relative to the pre-pairing baseline, EPSPs were enhanced 20-30 min following pairing in both control (one sample $t$-test, $t(7)=4.662, p=0.0023$ ) and CIE-exposed mice (one sample $t$-test 3 -day CIE, $t(9)=8.678, p<0.0001 ; 7$-day CIE, $t(8)=5.675, p=0.0005)$ with no difference in the magnitude of this between the groups. However, in air-exposed mice, EPSP amplitude returned to control levels and by 50-60 min, values were not different from the pre-STDP baseline (one sample $t$-test, $t(7)=0.5634, p=0.5907)$. In contrast, EPSP amplitude remained elevated in both groups of CIE-treated mice (one sample $t$-test 3 -day CIE, $t(9)=5.642, p=0.0003$; 7-day CIE, $t(8)=5.258, p=0.0008)$ and these values were significantly greater than the air control (one-way ANOVA: $\mathrm{F}_{(2,48)}=6.808, p=0.0025$; post-hoc comparison 3-day CIE: $q=4.594, p<0.0001$; 7 -day CIE: $q=4.286, p<0.001)$.

\section{WD from CIE Attenuates the Inhibitory Effect of Acute Ethanol Application on 10FC Neurons}

In this set of studies, we examined whether CIE treatment alters the inhibitory effect of acute ethanol on lOFC neuron excitability. Similar to that reported previously in alcoholnaive mice (Badanich et al, 2013), ethanol (33 mM) significantly reduced the number of evoked spikes from air control mice (two-way ANOVA: main effect of ethanol, $\mathrm{F}_{(1,7)}=6.66, p=0.036$; Figure 6a). Spiking was enhanced in neurons from CIE-treated mice with a near doubling in APs in the 3- (Figure 6b) and 7-day WD groups (Figure 6c; note different $y$ axis scales). In the 3-day WD group, ethanol had no effect on the number of APs induced during each current step (two-way ANOVA: $\mathrm{F}_{(1,6)}=1.14, p=0.327$; Figure $6 \mathrm{~b}$ ). In the 7-day (Figure 6c) and 10-day (Figure 6d) WD groups, there was also no significant effect of ethanol on AP number 


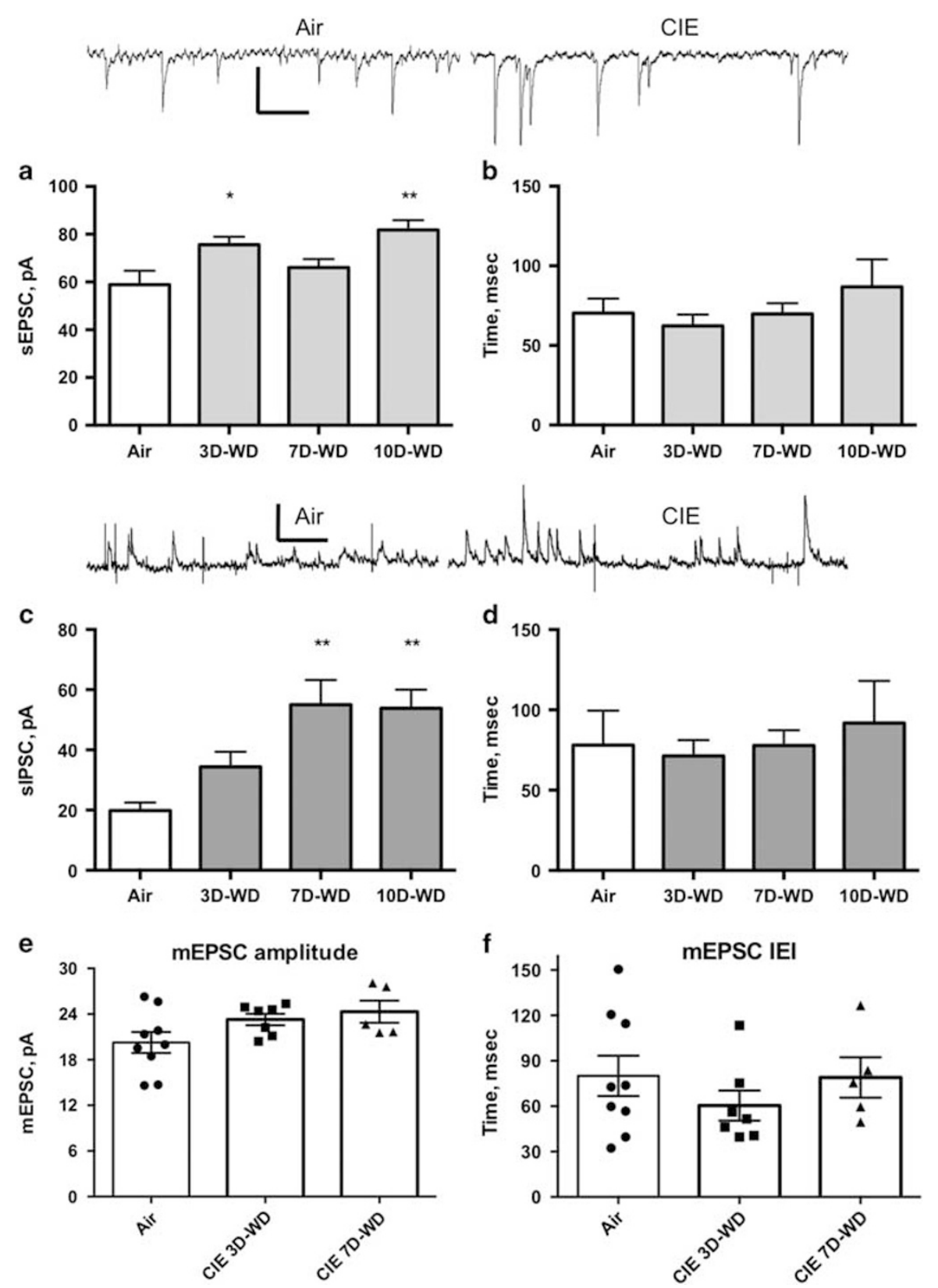

Figure 4 Chronic intermittent ethanol (CIE) exposure increases the amplitude, but not frequency (expressed as mean of the inter-event interval), of spontaneous excitatory postsynaptic currents (sEPSCs) and inhibitory postsynaptic currents (IPSCs) in lateral orbitofrontal cortex (IOFC) neurons. Representative traces of sEPSCs (top) and IPSCs (middle) from air- and CIE-treated (3D-WD) neurons are shown. Calibration bars $(x=50$ pA; $y=0.5 \mathrm{~s}$ ). A significant increase in amplitude (a; mean \pm SEM) but not frequency (b; expressed as inter-event interval) of AMPA-mediated sEPSCs was observed in CIEexposed mice (one-way analysis of variance (ANOVA): $F_{(3,39)}=2.889, p=0.048$ ) and pairwise comparisons to control revealed significant increases at the 3-day $(q=2.46, * p<0.05 ; n=17)$ and 10-day $(q=2.55$, $* * * 0.05 ; n=12)$ withdrawal (WD) time points. Increases in amplitude (c) but not frequency (d; expressed as inter-event interval) of sIPSCs were detected in 7-day $(q=3.74$, *** $p<0.01 ; n=20)$ and I0-day $(q=3.299$, ** $p<0.0$ I) WD animals. A trend toward an increase in the amplitude (e) but not frequency (f; expressed as inter-event interval) of miniature EPSCs (mEPSCs) was observed in CIE mice (oneway ANOVA: $\left.F_{(2,18)}=2.858, p=0.08\right)$, but pairwise comparisons revealed no significant differences between groups.

(7-day WD: two-way ANOVA, $\mathrm{F}_{(1,5)}=3.68, p=0.113 ; 10$ day WD: two-way ANOVA, $\left.\mathrm{F}_{(1,6)}=0.583, p=0.474\right)$

\section{WD from CIE does not Alter the Basal Activity of Extrasynaptic GABAA and Glycine Receptors of IOFC Neurons but Blunts the Ethanol-Induced Increase in Tonic Current}

Badanich et al (2013) reported that ethanol inhibition of IOFC neuron spike firing was mediated by an increase in a strychnine-sensitive current consistent with activation of glycine receptors. As intrinsic excitability can also be influenced by tonic currents carried by glycine or $\mathrm{GABA}_{\mathrm{A}}$ receptors, we examined whether CIE treatment alters these tonic inhibitory currents by measuring the holding current before and during local application of receptor blockers. As shown in Figure 7a, local application of gabazine induced small positive shifts in the holding currents of lOFC neurons in both air $(15.67 \pm 8.26 \mathrm{pA})$ and CIE groups (3-day WD: $10.48 \pm 11.03 \mathrm{pA}$; 7 -day WD: $20.04 \pm 26.7 \mathrm{pA})$, consistent 
a

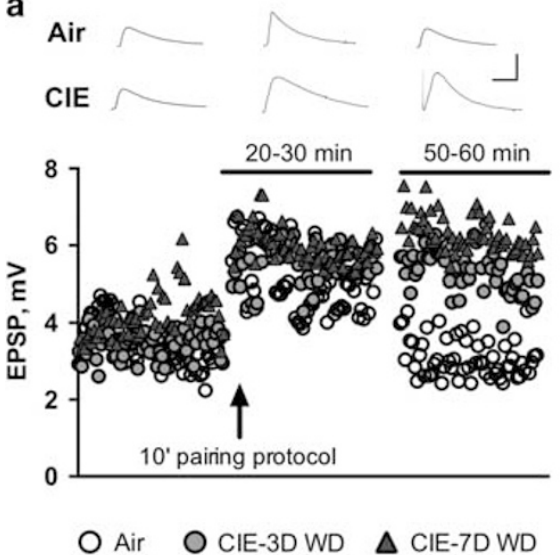

b
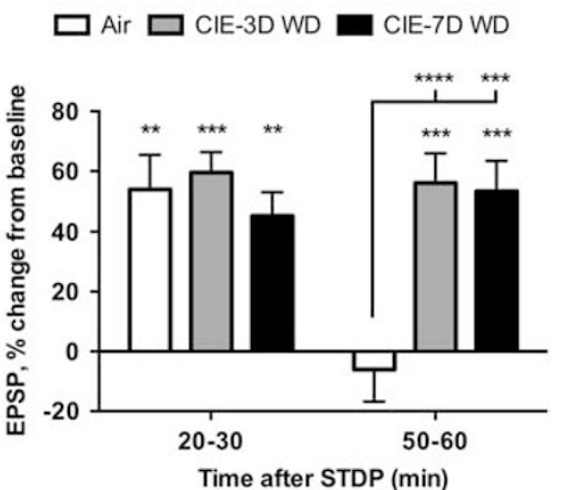

Figure 5 Chronic intermittent ethanol exposure (CIE) enhances the duration of glutamatergic synaptic plasticity induced by a spike-timing-dependent plasticity (STDP) protocol. (a) Representative traces showing stimulus-evoked AMPA EPSPs before (left) and after (20-30 min; middle; 50-60 min; right) spike-timing protocol. Calibration bars $(x=20 \mathrm{~ms} ; y=5 \mathrm{mV}$ ). Graphs show amplitude of mean EPSPs (SEM bars not shown for clarity) before and after STDP induction (arrow) for air- (open circles, $n=7$ ) and CIE-treated mice at 3-day (closed circles, $n=10$ ) and 7-day (closed triangles, $n=9$ ) after withdrawal $(W D$ ). (b) Significant increases in EPSP amplitude (values normalized to pre-pairing baseline; mean \pm SEM) at 20-30 min post pairing were observed in both control (one sample $t$-test vs I00\%, $t(7)=4.662$, *** $p=0.0023$ ) and ClE-exposed mice (one sample $t$-test vs I00\%, 3-day CIE, $t(9)=8.678$, *** $p<0.000$ I; 7-day CIE, $t(8)=5.675, * * * 0.0005)$ with no difference in the magnitude of this effect between groups. At 50-60 min post pairing, the increase in EPSP amplitude in ClE-exposed mice persisted (one sample $t$-test vs I00\%, 3-day CIE, $t(9)=5.642$, **** $p=0.0003 ; 7$-day CIE, $t(8)=5.258$, **** $p=0.0008$ ) and these values were significantly greater than the air control (one-way analysis of variance: $F_{(2,48)}=6.808, p=0.0025$; post-hoc comparison 3 -day $C I E$, $q=4.594$, ****** $p<0.000$; 7-day CIE, $q=4.286$, $* * * * * 0.00 \mathrm{I}$ ). The amplitude of EPSP at 50-60 min post pairing in air-exposed group returned to control levels that were not different from the pre-STDP baseline (one sample $t$-test, $t(7)=0.5634, p=0.5907$ ).

with the presence of a tonic $\mathrm{GABA}_{\mathrm{A}}$-mediated current. Analysis of this data revealed no differences in gabazinesensitive current between air controls and CIE-treated mice (one-way ANOVA: $\left.\mathrm{F}_{(2,28)}=0.115, p>0.05\right)$. Application of the glycine receptor antagonist strychnine also induced a small overall change in the holding current in both air control $(6.37 \pm 6.06 \mathrm{pA})$ and CIE-exposed mice (3-day WD: $9.06 \pm 8.96 \mathrm{pA}$; 7-day WD: $17.15 \pm 16.13 \mathrm{pA}$ ) (Figure 7b). These values were also not different from one another (oneway ANOVA: $\left.\mathrm{F}_{(2,30)}=0.3, p>0.05\right)$. To examine whether the loss of ethanol inhibition of spike firing following CIE treatment is associated with decreased expression of glycine receptors, western blotting was performed using an antibody that detects all four $\alpha$-subunits of the glycine receptor. GlyR immunoreactivity in $1 \mathrm{OFC}$ showed a trend toward an increase in CIE-exposed mice withdrawn for 3 days as compared with air controls; however, this difference was not statistically significant ( $t$-test, $t(8)=1.459, p=0.17)$ (Figure 7c). Although tonic glycine-mediated currents under baseline conditions were not altered by CIE treatment, results from Badanich et al (2013) showed that, in control animals, exposure to acute ethanol enhances these currents. To directly address whether the loss of ethanol modulation of spike firing in CIE-treated mice was due to an attenuation of this effect, we measured the shift in holding current during local application of acute ethanol to voltage-clamped OFC neurons. As shown in Figure 7d, ethanol produced a dosedependent and significant increase in the holding current in OFC neurons from air-treated mice. In contrast, no statistically significant change in holding current was found in neurons from CIE-treated mice withdrawn for 3 or 7 days. These findings suggest that mechanisms that regulate the extracellular levels of glycine may be altered by CIE treatment. To explore this further, we measured currentevoked spiking from control and CIE-treated neurons in the absence and presence of the GlyT1 inhibitor sarcosine. As shown in Figure $8 \mathrm{a}$, sarcosine $(100 \mu \mathrm{M})$ produced a significant inhibition of spike firing in OFC neurons from air-treated mice (two-way ANOVA: $\mathrm{F}_{(1,9)}=9.285$, $p=0.0139$ ). CIE treatment and WD enhanced spiking of lOFC neurons, consistent with data shown previously. However, sarcosine had no effect on current-evoked firing prepared from CIE mice at the 3- $\left(\mathrm{F}_{(1,5)}=1.177, p=0.327\right.$; Figure $8 b)$ or 7 -day $\left(\mathrm{F}_{(1,6)}=3.084, p=0.1296\right.$; Figure $\left.8 c\right) \mathrm{WD}$ period. To test whether the loss of sarcosine inhibition was associated with a reduction in GlyT1 expression, lOFC tissue from air- and CIE-treated mice was probed with an antiGlyT1 antibody. Western blotting revealed significantly reduced expression of GlyT1 in both the pellet and supernatant fractions of lOFC tissue (two-way ANOVA: $\left.\mathrm{F}_{(1,18)}=5.63, p=0.029\right)$ isolated from the 3-day CIE WD group (Figure 8d).

\section{DISCUSSION}

In this study, we show that repeated cycles of CIE exposure and WD in mice enhances the intrinsic excitability and synaptic properties of lOFC neurons. The robust increase in current-evoked AP firing in neurons from CIE-treated mice was not the result of changes in basal tonic currents induced by $\mathrm{GABA}_{\mathrm{A}}$ or glycine receptors, but was accompanied by a decrease in the functional activity of SK channels. CIEtreated mice also showed an increase in the AMPA/NMDA ratio that was associated with bidirectional changes in GluA1/A2 AMPA and GluN2B NMDA receptor protein expression. Neurons from CIE-exposed mice displayed a persistent LTP of glutamatergic synaptic transmission following a spike-timing protocol and AP spiking were markedly less sensitive to the inhibitory effect of acute 

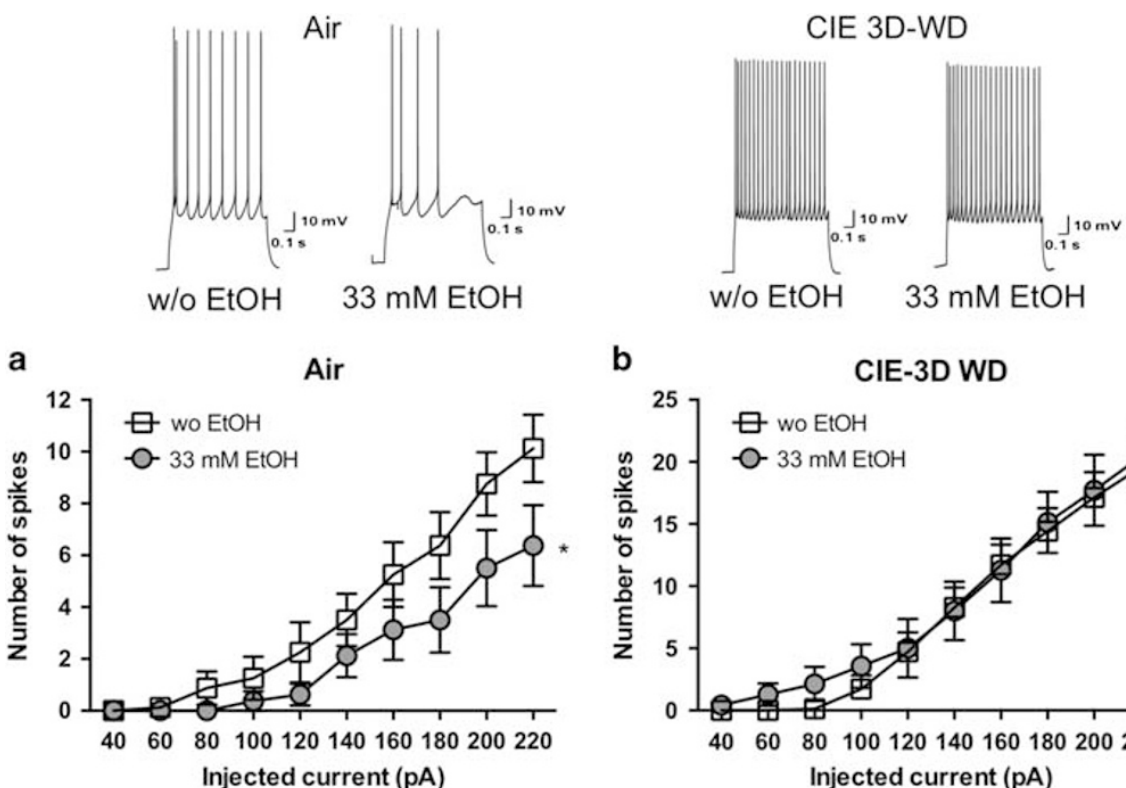

b
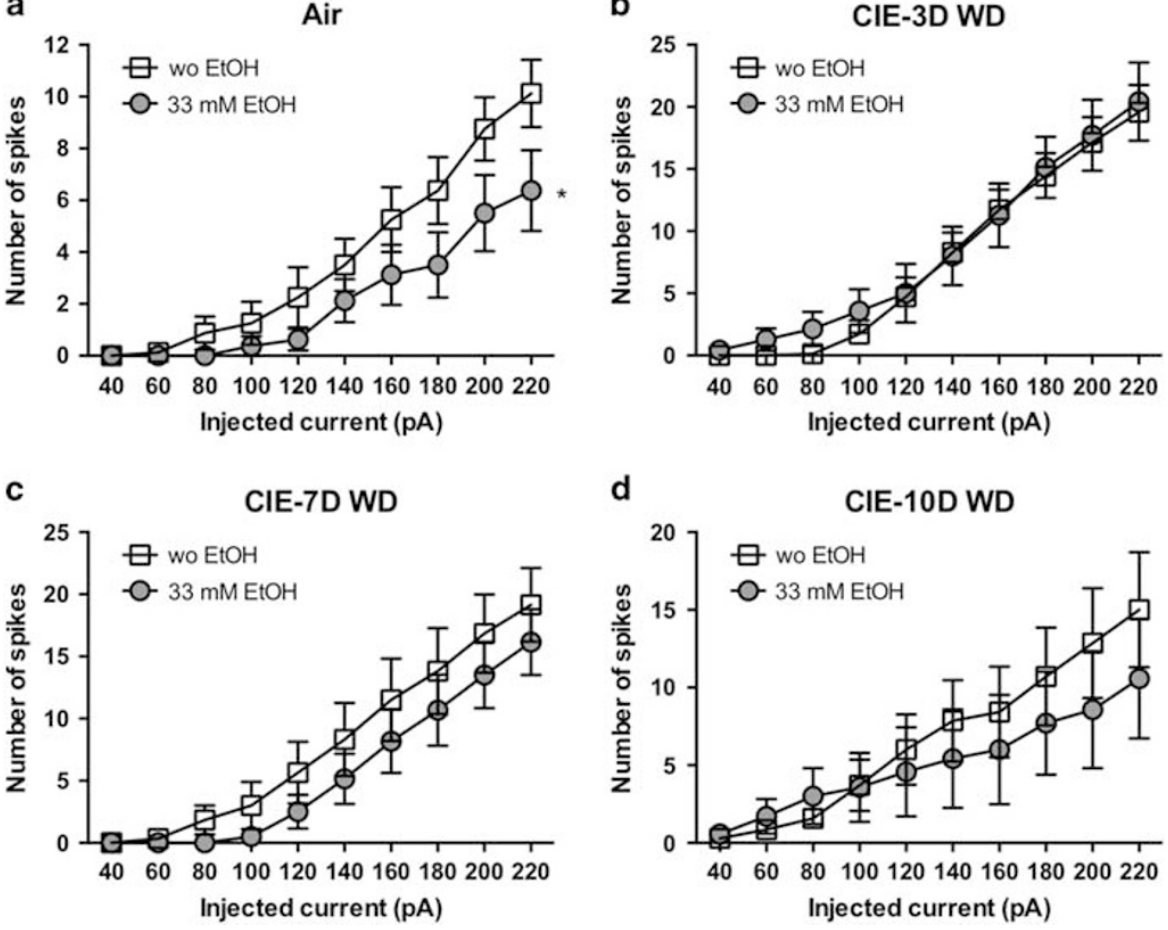

Figure 6 Chronic intermittent ethanol (CIE) exposure suppresses the ability of acute ethanol to inhibit spike firing of lateral orbitofrontal cortex (IOFC) neurons. Representative traces show current-evoked spiking in the absence and presence of $33 \mathrm{mM}$ ethanol applied to IOFC neurons from air control (left) and $\mathrm{ClE}$-treated (right) mice. Graphs show summary of the effect of acute ethanol ( $33 \mathrm{mM}, 10 \mathrm{~min}$ ) on action potential (AP) spiking (mean \pm SEM) induced by a series of current injections (40-220 pA) in IOFC neurons obtained from air control $(a, n=8)$ and CIE-treated mice at 3-day $(b, n=7), 7$-day $(c, n=6)$, and I0-days ( $d, n=7$ ) of withdrawal (WD). Similar to the results shown in Figure Ib, WD from CIE exposure produced an increase in AP firing (note different $y$ axes between $a$ and $b-e)$. Acute ethanol significantly reduced spiking in IOFC neurons obtained from air control (a; two-way analysis of variance (ANOVA): $F_{(I, 7)}=6.66$, $\left.{ }^{*} p=0.036\right)$, but did not significantly change AP firing in 3-day (b; two-way ANOVA: $\left.F_{(I, 6)}=1.14, p=0.327\right)$, 7-day (c; two-way ANOVA: $F_{(I, 5)}=3.68, p=0.113$ ) or I0-day (d; two-way ANOVA: $F_{(I, 6)}=0.583, p=0.474$ ) ClE groups.

ethanol and the GlyT1 inhibitor sarcosine. Taken together, these findings show that chronic exposure to ethanol causes multiple changes in OFC neuron function and suggests that these alterations may contribute to the impairment of OFCdependent behaviors often observed in alcohol-dependent individuals.

\section{CIE Increases Neuronal Excitability of 10FC Neurons and Reduces Sensitivity to Acute Ethanol}

A previous study from our laboratory demonstrated that ethanol, applied acutely, suppressed current-evoked AP firing in lOFC neurons (Badanich et al, 2013). This was accompanied by an increase in the holding current and both of these changes were blocked by strychnine, suggesting a role for glycine receptors in mediating ethanol action. In the present study, lOFC neuronal excitability was dramatically enhanced following exposure to repeated cycles of ethanol vapor and ethanol applied acutely no longer inhibited current-evoked spike firing. This was accompanied by a blunting of ethanol-induced increases in holding current observed in CIE-treated mice. The increase in currentevoked spiking and reduced ethanol sensitivity was observed at 3 days post $\mathrm{WD}$ and persisted for at least 10 days after the last ethanol exposure. The mechanism underlying these effects is not completely known but does not seem to involve a decrease in tonic currents mediated by glycine or $\mathrm{GABA}_{\mathrm{A}}$ receptor activity under baseline conditions, as these were essentially unchanged in CIE mice as compared with air controls. Western blotting with an antibody that recognizes all GlyR $\alpha$-subunits also failed to show any reduction in GlyR protein expression that might have been expected to underlie the loss of ethanol sensitivity. This suggests that CIE treatment may impair glycine release during acute ethanol exposure, or that glycine transporter function is enhanced in CIE-treated mice, thus shunting any ethanol-induced increase in extracellular glycine to levels below that needed to activate GlyRs. The former hypothesis seems more likely 

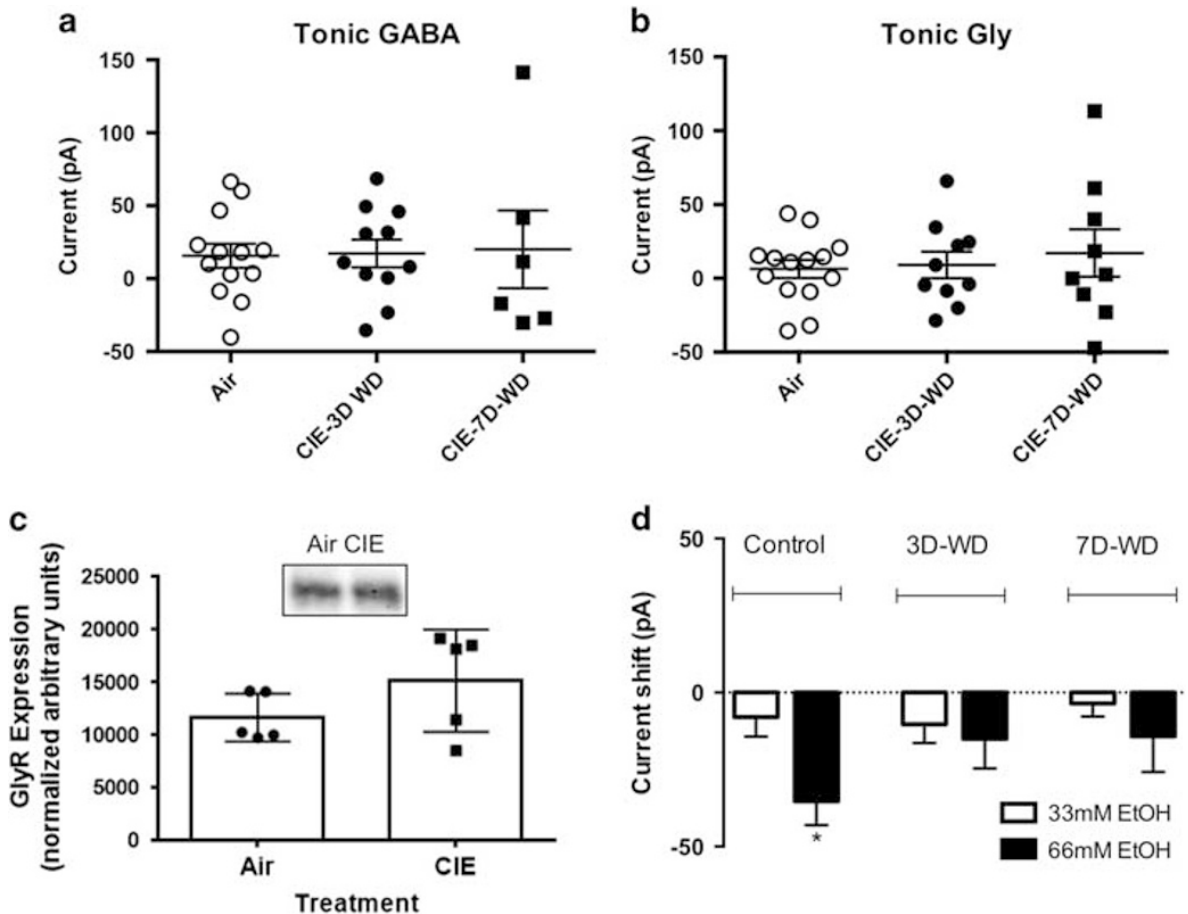

Figure 7 Chronic intermittent ethanol (CIE) exposure does not alter basal tonic currents mediated by GABA $\mathrm{A}_{\mathrm{A}}$ and glycine receptors in lateral orbitofrontal cortex (IOFC) neurons but blunts the ethanol-induced increase in holding current. Graphs show individual values and mean ( \pm SEM) changes in tonic current shift from baseline following application of gabazine (GABA $A$ currents; a) or gabazine plus strychnine (Glycine currents; b) in IOFC neurons obtained from air$(n=13)$ and CIE-treated mice at 3-day $(n=12)$ and 7-day $(n=6)$ after withdrawal (WD). No significant differences in the magnitude of basal tonic currents were found between air control and CIE groups (gabazine; one-way repeated-measures analysis of variance (ANOVA): $F_{(2,28)}=0.1$ I 5 , $p>0.05$; gabazine plus strychnine, one-way repeated-measures ANOVA: $\left.F_{(2,30)}=0.3, p>0.05\right)$. (c) Representative western blottings and optical density (mean \pm SEM) of glycine receptor $\alpha$-subunits in IOFC neurons from air and 3-day WD groups. There was no difference in expression of glycine receptors between air- and CIEexposed groups (two-tailed $t$-test, $t(8)=1.459, p=0.17$ ). (d) Mean changes in holding current in response to locally applied ethanol (33 and $66 \mathrm{mM}$ ) in IOFC neurons from air- $(n=12)$ and ClE-treated mice 3 days $(n=6)$ and 7 days $(n=7)$ after WD. Ethanol induced a significant increase in holding current in control (one-sample $t$-test, $t=4.57$, $*$ $p<0.0008$ ) but not ClE-treated neurons.

as the CIE-induced decrease in ethanol inhibition of spike firing and holding current was mirrored by a loss of sensitivity to sarcosine and reduced expression of the GlyT1 transporter.

The enhanced current-evoked firing of IOFC neurons was accompanied by a significant decrease in the AHP and a near total loss of apamin-sensitive currents, suggesting a downregulation of SK channel expression. Although the specific subtype of SK channels expressed by lOFC neurons is not yet known, no significant changes in apamin-sensitive SK subtypes (eg, SK2 and SK3) subunit expression were observed in CIE-treated mice. We note that the western blotting studies were carried out using tissue punches that included all layers of the OFC, and thus it is possible that a selective loss of SK channels in deep-layer pyramidal neurons of the lOFC may have been masked by opposing changes in other areas. However, a more parsimonious explanation is that CIE treatment induced a functional uncoupling of SK channels resulting in loss of at least one source of regulation of AP firing. Recent studies report either no change (Trantham-Davidson et al, 2014) or an increase (Pleil et al, 2015) in AP firing in prelimbic mPFC pyramidal neurons from CIE-exposed rats and mice, respectively. Increases in neuronal excitability following chronic exposure to ethanol have also been reported in other brain areas, including the VTA (Hopf et al, 2007), nucleus accumbens (Hopf et al, 2010), hippocampus (Mulholland et al, 2011), dorsal raphe
(Lowery-Gionta et al, 2014), and ventral BNST (Pleil et al, 2015). Several of these studies also reported decreases in SK channel function (Hopf et al, 2007, 2010; Mulholland et al, 2011; Padula et al, 2015), suggesting that these channels are particularly sensitive to chronic ethanol exposure. Although downregulation of SK channel function would be expected to alter AP firing, AHPs are also generated by KCNQ channels that mediate M-currents and $\mathrm{HCN}$ channels that underlie the $I_{\mathrm{H}}$ current (Gu et al, 2005; Hansen et al, 2008). Chronic ethanol exposure has been reported to alter the $I_{\mathrm{H}}$ current in VTA DA neurons (Okamoto et al, 2006), whereas changes in KCNQ expression or function following chronic ethanol exposure are less well studied. Future studies are needed to examine whether CIE exposure alters the ability of these channels to regulate the excitability of lOFC neurons.

\section{CIE and Glutamatergic Signaling}

CIE treatment resulted in a significant increase in the AMPA/NMDA ratio at the 3-day WD period. This change appeared to be mediated by opposing changes in AMPA and NMDA receptors with evidence for subtype-selective changes in subunit expression. AMPA receptors assemble from a family of four different subunits (GluA1-GluA4), whereas NMDA receptors require GluN1 and GluN2(A-D) subunits with GluN3(A-B) subunits producing subtle changes in receptor function (Traynelis et al, 2010). In the 


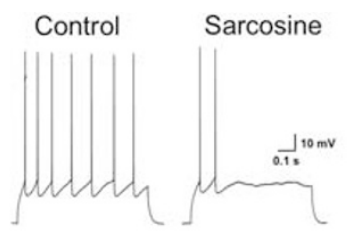

a

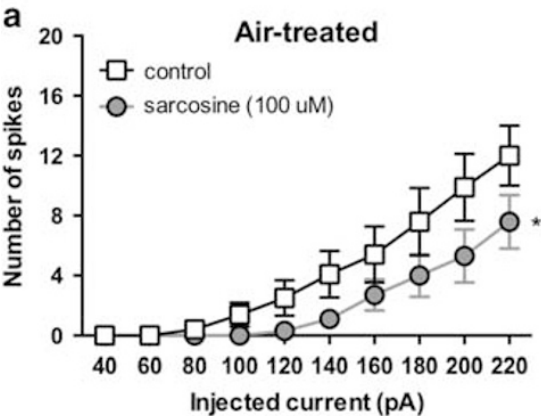

C

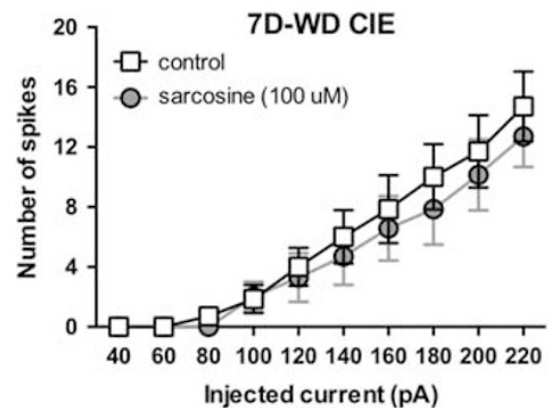

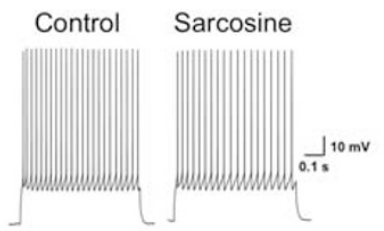

b

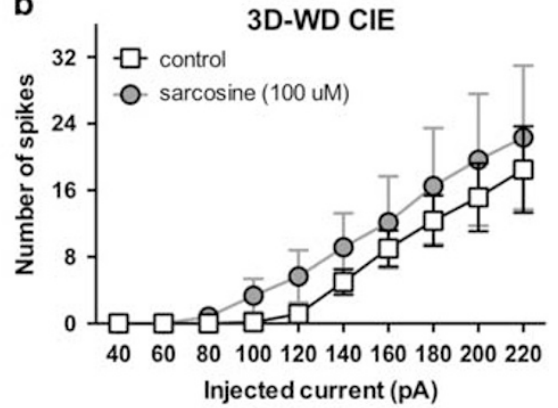

d

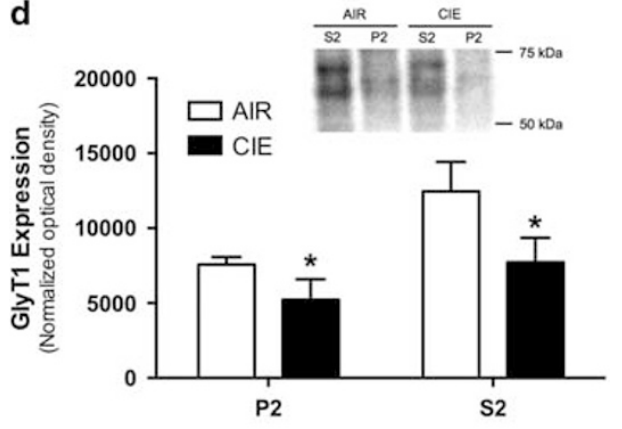

Figure 8 Chronic intermittent ethanol (CIE) exposure suppresses the ability of the GlyTI transport inhibitor sarcosine to reduce spiking in lateral orbitofrontal cortex (IOFC) neurons. Representative traces showing current-evoked spike firing in IOFC neurons from air-treated (left panel) or CIE-exposed mice (right panel) in the absence and presence of sarcosine (I00 $\mu \mathrm{M})$. Summary graphs show inhibitory effect of sarcosine (I00 $\mu \mathrm{M})$ on action potential spiking (mean \pm SEM) induced by a series of current injections (40-220 pA) in IOFC neurons obtained from air control (a, $n=10$, two-way analysis of variance (ANOVA): $F_{(I, 9)}=9.285$, * $\left.p=0.0139\right)$ but not ClE-treated mice tested at $3(b, n=6)$ and 7 days $(c, n=7)$. (d) ClE exposure reduced the expression of the Gly $T$ I transporter as measured by western blotting. Data are mean $( \pm$ SEM) of normalized optical density of Gly $T I$ immunoreactivity from air- $(n=5)$ and ClE-treated mice withdrawn for 3 days $(n=6)$. Two-way ANOVA shows significant effect of CIE treatment on $G$ ly $T I$ expression $\left(F_{(I, 18)}=5.63\right.$, * $\left.p=0.029\right)$ in pellet (P2) and supernatant (S2) fractions.

present study, GluN2B subunit expression was reduced in CIE-exposed mice with no change in GluN1 or GluN2A, while levels of GluA1 and GluA2 AMPA subunits were increased. The amplitude of evoked and spontaneous AMPA currents was also enhanced in CIE-exposed mice, while there was a trend toward a decrease in NMDA currents. These findings add to a growing understanding of ethanol-induced changes in glutamate receptor function and expression in the frontal cortex and confirm that such changes are both region and WD time dependent. For example, two previous studies from our group that used the same CIE protocol reported either no significant change in NMDA receptor expression in lOFC at 0 or 7 days post WD (McGuier et al, 2015), or an increase in GluN1 and GluN2B expression in the mPFC at the 0 but not 7 day WD time point (Kroener et al, 2012). A similar increase in GluN2B expression in the $\mathrm{mPFC}$ was reported for CIE-exposed rats killed $3 \mathrm{~h}$ after the last ethanol vapor treatment (Kim et al, 2014), while no change in GluN1 or GluN2(A/B) was noted 1 week post WD (TranthamDavidson et al, 2014), despite an increase in NMDAmediated EPSCs. In contrast, Holmes et al (2012) reported decreased GluN1 but not GluN2 expression in the mouse
$\mathrm{mPFC}$ at the 3-day WD period along with a reduction in evoked NMDA EPSCs in infralimbic mPFC neurons.

With regard to synaptic plasticity, in the present study, CIE treatment induced a sustained increase in glutamatergic signaling following a spike-timing dependent LTP protocol that was similar to that reported for deep-layer neurons in the prelimbic sub-division of the mPFC (Kroener et al, 2012). Although the cellular mechanisms underlying these changes are not completely understood, they likely arise from a combination of changes in factors that control intrinsic excitability and excitatory synaptic signaling. For example, the decrease in SK channel function following CIE treatment observed in many regions including the OFC would be expected to enhance synaptic efficacy and plasticity. In support of this, results from previous studies show that blocking these channels facilitates LTP and enhances learning and memory (Blank et al, 2003; Brennan et al, 2008; Hammond et al, 2006; Stackman et al, 2002), whereas overexpression of SK channels reduces LTP and impairs hippocampus-, amygdala-, and mPFC-dependent learning tasks. In addition to changes in glutamate receptor expression and function described above, alterations in dendritic 
structure and complexity of pyramidal neurons may also contribute to enhanced glutamatergic plasticity following ethanol exposure. Although not examined in the present study, our previous report showed that CIE exposure increased spine density in lOFC neurons due to an increase in highly plastic long thin spines (McGuier et al, 2015). Changes in spine density and/or subtype have also been reported in $\mathrm{MPFC}$ neurons from CIE-treated mice (Kroener et al, 2012) and rats (Kim et al, 2014), whereas an increase in apical dendrite length of prelimbic mPFC neurons has been observed in CIE-exposed mice (Holmes et al, 2012).

Overall, these findings, although at times appearing discordant, suggest that neurons within discrete areas of the PFC/OFC undergo dramatic and rapid shifts in excitability, synaptic drive, and morphology during the hours and days following WD from CIE treatment, with the direction and magnitude of the change being critically dependent on the time point and sub-region examined. Although not yet completely understood, these dynamic changes in PFC/OFC neuronal function may contribute to the alterations in OFC- and PFC-based behaviors (eg, reversal learning and set-shifting) that are observed during post-WD periods similar to those examined in this study (Badanich et al, 2011; DePoy et al, 2013; Holmes et al, 2012; Kroener et al, 2012; Trantham-Davidson et al, 2014). Such changes, if present in alcohol-dependent humans, may contribute to cognitive and behavioral deficits associated with long-term alcohol abuse that underlie the inability of these individuals to exert cognitive control over their drinking in the face of adverse consequences.

\section{Summary}

Overall, the results of this study suggest that chronic ethanol exposure leads to enhanced intrinsic excitability and glutamatergic synaptic signaling of lOFC neurons and blunted responsiveness to acute ethanol. These alterations may contribute to the alterations in OFC-dependent behaviors observed in previous studies and may contribute to the escalation in drinking associated with chronic intermittent exposure to ethanol. Knowledge of the fundamental properties of OFC neurons in response to chronic ethanol exposure may lead to better understanding of the pathology and treatment of alcohol-dependent individuals.

\section{FUNDING AND DISCLOSURE}

The authors declare no conflict of interest.

\section{ACKNOWLEDGMENTS}

We thank Diana Fulmer for assistance with the western blotting studies and Laura Ralston for coordinating the vapor inhalation treatments. This work was supported by P50AA010761 and R37AA009986 to JJW, UO1 AA020929 to MFL, and U01AA020930 to PJM.

\section{REFERENCES}

Aldridge GM, Podrebarac DM, Greenough WT, Weiler IJ (2008). The use of total protein stains as loading controls: an alternative to high-abundance single-protein controls in semi-quantitative immunoblotting. J Neurosci Methods 172: 250-254.

Badanich K, Becker H, Woodward J (2011). Effects of chronic intermittent ethanol exposure on orbitofrontal and medial prefrontal cortex-dependent behaviors in mice. Behav Neurosci 125: 879-891.

Badanich K, Mulholland P, Beckley J, Trantham-Davidson H, Woodward J (2013). Ethanol reduces neuronal excitability of lateral orbitofrontal cortex neurons via a glycine receptor dependent mechanism. Neuropsycopharmacology 38: 1176-1188.

Bechara A (2005). Decision making, impulse control and loss of willpower to resist drugs: a neurocognitive perspective. Nat Neurosci 8: 1458-1463.

Bechara A, Van Der Linden M (2005). Decision-making and impulse control after frontal lobe injuries. Curr Opin Neurol 18: 734-739.

Bissonette G, Martins G, Frans T, Harper E, Schoenbaum G, Powell E (2008). Double dissociation of the effects of medial and orbital prefrontal cortical lesions on attentional and affective shifts in mice. J Neurosci 28: 11124-11130.

Bissonette GB, Schoenbaum G, Roesch MR, Powell EM (2015). Interneurons are necessary for coordinated activity during reversal learning in orbitofrontal cortex. Biol Psychiatry 77: 454-464.

Blank T, Nijholt I, Kye M, Radulovic J, Spiess J (2003). Smallconductance, $\mathrm{Ca} 2+$-activated $\mathrm{K}+$ channel SK3 generates agerelated memory and LTP deficits. Nat Neurosci 6: 911-912.

Bokura H, Yamaguchi S, Kobayashi S (2001). Electrophysiological correlates for response inhibition in a Go/NoGo task. Clin Neurophysiol 112: 2224-2232.

Brennan A, Dolinsky B, Vu M, Stanley M, Yeckel M, Arnsten A (2008). Blockade of IP3-mediated SK channel signaling in the rat medial prefrontal cortex improves spatial working memory. Learn Mem 15: 93-96.

Brodie M, Appel S (1998). The effects of ethanol on dopaminergic neurons of the ventral tegmental area studied with intracellular recording in brain slices. Alcohol Clin Exp Res 22: 236-244.

Brodie M, McElvain M, Bunney E, Appel S (1999). Pharmacological reduction of small conductance calcium-activated potassium current (SK) potentiates the excitatory effect of ethanol on ventral tegmental area dopamine neurons. J Pharmacol Exp Ther 290: 325-333.

Brown K, Calizo L, Goodlett C, Staton M (2007). Neonatal alcohol exposure impairs acquisition of eyeblink conditioned responses during discrimination learning and reversal in weanling rats. Dev Psychobiol 49: 243-257.

DePoy L, Daut R, Brigman J, MacPherson K, Crowley N, Gunduz-Cinar O et al (2013). Chronic alcohol produces neuroadaptations to prime dorsal striatal learning. Proc Natl Acad Sci USA 110: 14783-14788.

Dias R, Robbins T, Roberts A (1996). Dissociation in prefrontal cortex of affective and attentional shifts. Nature 380: 69-72.

Dittmer A, Dittmer J (2006). Beta-actin is not a reliable loading control in Western blot analysis. Electrophoresis 27: 2844-2845.

Fortier C, Maksimovskiy A, Venne J, Lafleche G, McGlinchey R (2009). Silent trace eliminates differential eyeblink learning in abstinent alcoholics. Int J Environ Res Public Health 6: 2007-2027.

Fortier C, Steffen E, Lafleche G, Venne J, Disterhoft J, McGlinchey R (2008). Delay discrimination and reversal eyeblink classical conditioning in abstinent chronic alcoholics. Neuropsychology 22: 196-208.

Goldstein R, Tomasi D, Rajaram S, Cottone LA, Zhang L, Maloney $\mathrm{T}$ et al (2007). Role of the anterior cingulate and medial orbitofrontal cortex in processing drug cues in cocaine addiction. Neuroscience 144: 1153-1159.

Goldstein R, Volkow N (2002). Drug addiction and its underlying neurobiological basis: neuroimaging evidence for the involvement of the frontal cortex. Am J Psychiatry 159: 1642-1652. 
Gu N, Vervaeke K, Hu H, Storm J (2005). Kv7/KCNQ/M and HCN/ $\mathrm{h}$, but not KCa2/SK channels, contribute to the somatic medium after-hyperpolarization and excitability control in CA1 hippocampal pyramidal cells. J Physiol 556.3: 689-715.

Hammond R, Bond C, Strassmaier T, Ngo-Anh T, Adelman J, Maylie J et al (2006). Small-conductance Ca2+-activated $\mathrm{K}+$ channel type 2 (SK2) modulates hippocampal learning, memory, and synaptic plasticity. J Neurosci 26: 1844-1853.

Hansen H, Waroux O, Seutin V, Jentsch T, Aznar S, Mikkelsen J (2008). Kv7 channels: interaction with dopaminergic and serotoninergic neurotransmission in the CNS. J Physiol 586.7: $1823-1832$.

Holmes A, Fitzgerald PJ, MacPherson KP, DeBrouse L, Colacicco G, Flynn SM et al (2012). Chronic alcohol remodels prefrontal neurons and disrupts NMDAR-mediated fear extinction encoding. Nat Neurosci 15: 1359-1361.

Hopf F, Bowers M, Chang SJ, Chen B, Martin M, Seif T et al (2010). Reduced nucleus accumbens SK channel activity enhances alcohol seeking during abstinence. Neuron 65: 682-694.

Hopf F, Martin M, Chen B, Bowers M, Mohamedi M, Bonci A (2007). Withdrawal from intermittent ethanol exposure increases probability of burst firing in VTA neurons in virto. J Neurophysiol 98: 2297-2310.

Iversen S, Mishkin M (1970). Perseverative interference in monkeys following selecetive lesions of the inferior prefrontal convexity. Exp Brain Res 11: 376-386.

Jedema H, Carter M, Dugan B, Gurnsey K, Olsen A, Bradberry C (2011). Acute impact of ethanol on cognitive performance in rhesus macaques. Cerebral cortex. Cereb Cortex 21: 1783-1791.

Jentsch J, Olausson P, De La Garza R 2nd, Taylor J (2002). Impairments of reversal learning and response perseveration after repeated, intermittent cocaine administrations to monkeys. Neuropsycopharmacology 26: 183-190.

Kaczorowski CC, Disterhoft J, Spruston N (2007). Stability and plasticity of intrinsic membrane properties in hippocampal CA1 pyramidal neurons: effects of internal anions. J Physiol 578: 799-818.

Kay AR (1992). An intracellular medium formulary. J Neurosci Methods 44: 91-100.

Kim A, Zamora-Martinez ER, Edwards S, Mandyam CD (2014). Structural reorganization of pyramidal neurons in the medial prefrontal cortex of alcohol dependent rats is associated with altered glial plasticity. Brain Struct Funct 220: 1705-1720.

Kolb B, Pellis S, Robinson T (2004). Self administration of cocaine modifies neuronal morphology in nucleus accumbens and prefrontal cortex. Brain Cogn 55: 104-115.

Kringelbach M, Rolls E (2004). The functional neuroanatomy of the human orbitofrontal cortex: evidence from neuroimaging and neuropsychology. Prog Neurobiol 72: 341-372.

Kroener S, Mulholland P, New N, Gass J, Becker H, Chandler L (2012). Chronic alcohol exposure alters behavioral and synaptic plasticity of the rodent prefrontal cortex. PLoS One 7: e37541.

Lopez de Armentia M, Sah P (2004). Firing properties and connectivity of neurons in the rat lateral central nucleus of the amygdala. J Neurophysiol 92: 1285-1294.

Lowery-Gionta E, Marcinkiewcz C, Kash TL (2014). Functional alterations in the dorsal raphe nucleus following acute and chronic ethanol exposure. Neuropsycopharmacology 40: 590-600.

Ma Y, Hemley S, Toll J, Jentsch J, Evans C, Levine M et al (2013). Drug-primed reinstatement of cocaine seeking in mice: increased excitability of medium-sized spiny neurons in the nucleus accumbens. ASN Neuro 5: 257-271.

Matochik J, London E, Eldreth D, Cadet J, Bolla K (2003). Frontal cortical tissue composition in abstinent cocaine abusers: a magnetic resonance imaging study. Neuroimage 19: 1095-1102.

McAlonan K, Brown V (2003). Orbital prefrontal cortex mediates reversal learning and not attentional set shhifting in the rat. Behav Brain Res 146: 97-103.
McGuier N, Padula A, Lopez M, Woodward J, Mulholland P (2015). Withdrawal from chronic intermittent alcohol exposure increases dendritic spine density in the lateral orbitofrontal cortex in mice. Alcohol 49: 21-27.

Mulholland P, Becker H, Woodward J, Chandler L (2011). SK2 channels regulate alcohol-associated plasticity of glutamatergic synapses. Biol Psychiatry 69: 625-632.

O'Doherty J, Kringelbach M, Rolls E, Hornak J, Andrews C (2001). Abstract reward and punishment representations in the human orbitofrontal cortex. Nat Neurosci 4: 95-102.

Okamoto T, Harnett M, Morikawa H (2006). Hyperpolarizationactivated cation current ( $\mathrm{Ih}$ ) is an ethanol target in midbrain dopamine neurons of mice. J Neurophysiol 95: 619-626.

Padula AE, Griffin WC 3rd, Lopez MF, Nimitvilai S, Cannady R, McGuier NS et al (2015). KCNN genes that encode small-conductance $\mathrm{Ca}(2+)$-activated $\mathrm{K}(+)$ channels influence alcohol and drug addiction. Neuropsychopharmacology 40: 1928-1939.

Pleil KE, Lowery-Gionta EG, Crowley NA, Li C, Marcinkiewcz CA, Rose JH et al (2015). Effects of chronic ethanol exposure on neuronal function in the prefrontal cortex and extended amygdala. Neuropharmacology (e-pub ahead of print) 10.1016.

Ponomarev I, Wang S, Zhang L, Harris RA, Mayfield RD (2012). Gene coexpression networks in human brain identify epigenetic modifications in alcohol dependence. J Neurosci 32: 1884-1897.

Ramus S, Eichenbaum H (2000). Neural correlates of olfactory recognition memory in the rat orbitofrontal cortex. J Neurosci 20: 8199-8208.

Robinson T, Gorny G, Mitton E, Kolb B (2001). Cocaine selfadministration alters the morphology of dendrites and dendritic spines in the nucleus accumbens and neocortex. Synapse 39: 257-266.

Robinson T, Kolb B (1999). Alterations in the morphology of dendrites and dendritic spines in the nucleus accumbens and prefrontal cortex following repeated treatment with amphetamine or cocaine. Eur J Neurosci 11: 1598-1604.

Roesch M, Calu D, Burke K, Schoenbaum G (2007a). Should I stay or should I go? Transformation of time-discounted rewards in orbitofrontal cortex and associated brain circuits. Ann N Y Aced Sci 1104: 21-34.

Roesch M, Stalnaker T, Schoenbaum G (2007b). Associative encoding in anterior piriformis cortex versus orbitofrontal cortex during odor discrimination and reversal learning. Cereb Cortex 17: $643-652$

Rogers R, Everitt B, Baldacchino A, Blackshaw A, Swaninson R, Wynne $\mathrm{K}$ et al (1999a). Dissociable deficits in the decisionmaking cognition of chronic amphetamine abusers, opiate abusers, patients with focal damage to prefrontal cortex, and tryptophan-depleted normal volunteers: Evidence for monoaminergic mechanisms. Neuropsycopharmacology 20: 322-339.

Rogers R, Owen A, Middleton H, Williams E, Pickard J, Sahakian B et al (1999b). Choosing between small, likely rewards and large, unlikely rewards activates inferior and orbital prefrontal cortex. J Neurosci 19: 9029-9038.

Sah P (1996). Ca(2+)-activated $\mathrm{K}+$ currents in neurones: types, physiological roles and modulation. Trends Neurosci 19: 150-154.

Sah P, Faber E (2002). Channels underlying neuronal calciumactivated potassium currents. Prog Neurobiol 66: 345-353.

Sailer C, Hu H, Kaufmann W, Trieb M, Schwarzer C, Storm J et al (2002). Regional differences in distribution and functional expression of small-conductance $\mathrm{Ca} 2+$-activated $\mathrm{K}+$ channels in rat brain. J Neurosci 22: 9698-9707.

Sailer C, Kaufmann W, Marksteiner J, Knaus H (2004). Comparative immunohistochemical distribution of three smallconductance $\mathrm{Ca} 2+$-activated potassium channel subunits, SK1, SK2, and SK3 in mouse brain. Mol Cell Neurosci 26: 458-469. 
Schoenbaum G, Chiba A, Gallagher M (1998). Orbitofrontal cortex and basolateral amygdala encode expected outcomes during learning. Nat Neurosci 1: 155-159.

Schoenbaum G, Saddoris M, Ramus S, Shaham Y, Selow B (2004). Cocaine-experienced rats exhibit learning deficits in a task sensitive to orbitofrontal cortex lesions. Eur J Neurosci 19: 1997-2002.

Stackman R, Hammond R, Linardatos E, Gerlach A, Maylie J, Adelman J et al (2002). Small conductance Ca2+-activated K+ channels modulate synaptic plasticity and memory encoding. J Neurosci 22: 10163-10171.

Thorpe S, Rolls E, Maddison S (1983). The orbitofrontal cortex: neuronal activity in the behaving monkey. Exp Brain Res 49: 93-115.

Trantham-Davidson H, Burnett EJ, Gass JT, Lopez MF, Mulholland PJ, Centanni SW et al (2014). Chronic alcohol disrupts dopamine receptor activity and the cognitive function of the medial prefrontal cortex. J Neurosci 34: 3706-3718.

Traynelis SF, Wollmuth LP, McBain CJ, Menniti FS, Vance KM, Ogden KK et al (2010). Glutamate receptor ion channels: structure, regulation, and function. Pharmacol Rev 62: 405-496.
Tremblay L, Schultz W (1999). Relative reward preference in primate orbitofrontal cortex. Nature 398: 704-708.

Velumian A, Zhang L, Pennefather P, Carlen P (1997). Reversible inhibition of IK, IAHP, Ih and ICa currents by internally applied gluconate in rat hippocampal pyramidal neurones. Pflugers Arch 433: 343-350.

Verdejo-Garcia A, Bechara A, Recknor E, Perex-Garcia M (2006). Executive dysfunction in substance dependent individuals during drug use and abstinence: an examination of the behavioral, cognitive and emotional correlates of addiction. J Int Neuropsychol Soc 12: 405-415.

Villalobos C, Shakkottai V, Chandy KG, Michelhaugh S, Andrade R (2004). SKCa channels mediate the medium but not the slow calcium-activated afterhyperpolarization in cortical neurons. $J$ Neurosci 24: 3537-3542.

Volkow N, Fowloer J, Wang GJ (2004). The addicted human brain viewed in the light of imaging studies: brain circuits and treatment strategies. Neuropharmacology 47: 3-13.

Zhang L, Weiner J, Valiante T, Velumian A, Watson P, Jahromi S et al (1994). Whole-cell recording of the $\mathrm{Ca}(2+)$-dependent slow afterhyperpolarization in hippocampal neurones: efffects of internally applied anions. Pflugers Arch 426: 247-253.

Supplementary Information accompanies the paper on the Neuropsychopharmacology website (http://www.nature.com/npp) 